\title{
Symplectic Lefschetz fibrations of curves as gonal coverings
}

\author{
Jaume Amorós · Volker Braungardt · Javier Vindel
}

Received: 14 March 2012 / Accepted: 8 November 2012 / Published online: 21 November 2012

(C) Springer Science+Business Media Dordrecht 2012

\begin{abstract}
We show that after a finite base change every symplectic Lefschetz fibration $f: X \rightarrow B$ of genus $g>3$ curves over a closed oriented surface becomes a finite covering of degree $\frac{g}{2}+1$ or $\frac{g}{2}+\frac{3}{2}$ of a family of spheres over a Riemann surface, with a branch locus admitting complex algebraic curves as local models. In the case of fibers of genus 4 , it is shown that after a 2:1 base change the family admits a trigonal covering to a symplectic ruled surface, with symplectic branch locus.
\end{abstract}

Keywords Symplectic 4-manifolds · Lefschetz fibrations · Hurwitz spaces ·

Trigonal coverings

\section{Mathematics Subject Classification $\quad$ 53D35 $57 \mathrm{R} 17 \cdot 14 \mathrm{H} 45 \cdot 14 \mathrm{D} 05 \cdot 14 \mathrm{D} 22$}

\section{Contents}

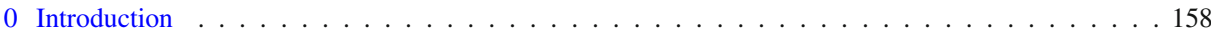

1 Compatible symplectic structures in a 4-dimensional Lefschetz fibration . . . . . . . . . . . . . . 160

2 Almost complex structures . . . . . . . . . . . . . . . . . . . . . 161

2.1 Transversality properties of compatible almost complex structures . . . . . . . . . . . . 161

2.2 Transversality to the orbifold locus in $\overline{\mathcal{M}}_{g} \ldots \ldots \ldots \ldots \ldots \ldots$

3 Polygonality . . . . . . . . . . . . . . . . . . . . . . . 167

3.1 Hurwitz spaces of adequate dimension . . . . . . . . . . . . . . . . . . . . . 167

3.2 Global polygonal structure . . . . . . . . . . . . . . . . . . . . . 168

4 Boundary components of Hurwitz space . . . . . . . . . . . . . . . . . . . . . . 171

Research originally supported by EDGE, Research Training Network HPRN-CT-2000-00101, supported by The European Human Potential Programme, and by the Deutsche Forschungsgemeinschaft. Concluded under support from grants MTM2009-14163-C02-02, from the Kingdom of Spain, and 2009 SGR 1284, from Catalonia.

J. Amorós $(\varangle) \cdot$ V. Braungardt · J. Vindel

Departament de Matemàtica Aplicada I, Universitat Politècnica de Catalunya, Diagonal 647, 08028 Barcelona, Spain

e-mail: jaume.amoros@upc.edu 
4.1 Reducible fibres . . . . . . . . . . . . . . . . . . . . . . . . . 171

4.2 Irreducible singular fibres . . . . . . . . . . . . . . . . . . . . . . . . 172

4.3 Regular fibres . . . . . . . . . . . . . . . . . . . . . . . . . . . . 173

5 Smoothness of the Hurwitz space . . . . . . . . . . . . . . . . . . . . . . . . . . . . . 174

6 Genus 4 fibrations . . . . . . . . . . . . . . . . . . . . . . . . . . . . 177

\section{Introduction}

This work is a study on the topology of closed symplectic 4-manifolds, motivated by the analysis of Lefschetz pencils and fibrations with hyperelliptic fibers in [15].

In that reference, the authors show how to glue the hyperelliptic structure in the fibers of a Lefschetz fibration $f: X \rightarrow S^{2}$ to achieve a fibration which is globally hyperelliptic, that is, after some auxiliary blow up in finitely many points $\sigma: \tilde{X} \rightarrow X$ the family becomes a double cover $\kappa: \tilde{X} \rightarrow P$, where $P$ is a symplectic ruled surface, i.e. a symplectic fibration over $S^{2}$ with fibers again 2-spheres, and the 2:1 map $\kappa$ becomes a branched cover, with its ramification set $R \subset P$ a symplectic submanifold, locally modelled by complex algebraic curves.

Siebert and Tian also show in [15] that, in order for a Lefschetz fibration of curves to admit a hyperelliptic structure in all its fibers, the geometric monodromy of the fibration must lie within the hyperelliptic mapping class group, which is much smaller than the general mapping class group. This is a very restrictive topological condition, except in the case when the fibers have genus $g=2$ because then they are all hyperelliptic. This latter fact was used in $[2,16]$ to achieve a thorough comparison of symplectic and holomorphic Lefschetz fibrations of curves of genus 2, while in the case of genus $g>2$ the hyperelliptic monodromy restriction has been too strong to allow its application to the study of general Lefschetz pencils.

The aim of this manuscript is to continue the study of general Lefschetz fibrations with fibers of genus $g>3$, and their comparison to holomorphic fibrations, using coverings with the generic gonality in each genus. The classical analysis of linear series in complex algebraic curves based on the Riemann-Roch theorem (see [3, Ch. IV]) shows that

- for even genus $g$, all curves admit a holomorphic cover $C_{g} \rightarrow \mathbb{P}_{\mathbb{C}}^{1}$ of degree $\frac{g}{2}+1$, and a generic $C_{g}$ admits only finitely many such covers,

- for odd genus $g$, all curves admit a holomorphic cover $C_{g} \rightarrow \mathbb{P}_{\mathbb{C}}^{1}$ of degree $\frac{g}{2}+\frac{3}{2}$, and a generic $C_{g}$ admits a 1-dimensional family of such covers,

- for degrees $d$ smaller than $\frac{g}{2}+1$ for even $g, \frac{g}{2}+\frac{3}{2}$ for odd $g$, only special curves (i.e., a complex subvariety in moduli space $\mathcal{M}_{g}$ ) admit maps to $\mathbb{P}_{\mathbb{C}}^{1}$ of degree $d$.

The case of fibrations with fiber genus $g=3$ is left out of this work because of the complications posed by the hyperelliptic divisor in the moduli space of curves $\overline{\mathcal{M}}_{3}$.

For a symplectic 4-manifold $(X, \omega)$ and Lefschetz fibration $f: X \rightarrow B$ with fibers of genus $g>3$, the above analysis is applied in this work in the following way:

First, we show in Proposition 4 that for a given Lefschetz fibration $f: X \rightarrow B$, the space of symplectic structures on $X$ compatible with the fibration is arc-connected.

Next, we discuss in Sect. 2 the existence of almost complex structures $J$ on $X$ compatible with the Lefschetz fibration, i.e. such that all fibers become complex algebraic curves, and we show in Corollary 9, Proposition 11 that a generic such $J$ defines a smooth classifying map to the moduli space of curves $\varphi: B \rightarrow \overline{\mathcal{M}}_{g}$ which is transverse to any desired finite set of subvarieties (formed by curves with automorphisms, special linear series ...) and the unicity up to deformation of such compatible, transverse almost complex structures $J$.

Having established the existence of compatible almost complex structures $J$ such that the induced classifying map $\varphi$ avoids, by transversality, any behaviour of the fibers that is found 
in a subvariety of codimension $>1$ in $\overline{\mathcal{M}}_{g}$, we turn to Algebraic Geometry to construct the ramified covers and to determine which "singular" behaviours of the fibers and covers will be found in divisors of $\overline{\mathcal{M}}_{g}$, thus will be a priori unavoidable in our symplectic fibrations.

In Sect. 3, we first discuss in Sect. 3.1 which is the suitable space of covers of $\mathbb{P}_{\mathbb{C}}^{1}$ such that it admits a finite map $\tilde{H} \rightarrow \overline{\mathcal{M}}_{g}$. For even genus $g$ this is just the stable completion of Hurwitz space $\tilde{H}=\overline{\mathcal{H}}_{d, g}$, with degree $d=\frac{g}{2}+1$ (see $[6,9,14]$ ). The solution for odd genus $g$ is more cumbersome: the first Hurwitz space covering $\overline{\mathcal{M}}_{g}$ is that with degree of the cover $d=\frac{g}{2}+\frac{3}{2}$, but the moduli map $\overline{\mathcal{H}}_{d, g} \rightarrow \overline{\mathcal{M}}_{g}$ now has 1-dimensional fibers. We fix as a level structure for the Hurwitz covers the requirement that one of the cross ratios of the branch points has a fixed value $\varepsilon$, and this defines a divisor $H \subset \overline{\mathcal{H}}_{d, g}$ with a finite moduli map. The space of coverings $\tilde{H}$ is then the normalization of $H$, and we prove in Corollary 14 that it has the property that all subvarieties $S \subset \overline{\mathcal{H}}_{d, g}$ defined by a topological type of admissible coverings intersect $H$ with the same codimension as they have in $\overline{\mathcal{H}}_{d, g}$.

Once a space of covers $\tilde{H}$ with a finite moduli map $h: \tilde{H} \rightarrow \overline{\mathcal{M}}_{g}$ has been found, the classifying map $\varphi: B \rightarrow \overline{\mathcal{M}}_{g}$ defined by a compatible almost complex structure in a symplectic Lefschetz fibration $f: X \rightarrow B$ can be pulled back by $h$, and this defines a new symplectic Lefschetz fibration $\tilde{f}: \tilde{X} \rightarrow \tilde{B}=h^{*} B$, with $\tilde{B}$ a closed surface, and $\tilde{X} \rightarrow X$ a finite cover branched over finitely many fibers. We establish in Theorem 15 that the compatible almost complex structure $J$ on $X \rightarrow B$ may be selected to achieve transversality to the image of the divisors from $\partial \mathcal{H}_{d, g}$ in $\overline{\mathcal{M}}_{g}$, and this determines the intersection with these divisors of the pulled back classifying map $\Phi: \tilde{B} \rightarrow \overline{\mathcal{H}}_{d, g}$.

The pulled back fibration $\tilde{f}: \tilde{X} \rightarrow \tilde{B}$ has become, besides a symplectic Lefschetz fibration, a family of degree $d$ branched covers $X_{s} \rightarrow \mathbb{P}_{\mathbb{C}}^{1}$.

In Sect. 4 we determine all the topological types of $d$-gonal coverings of the Riemann sphere which are projected onto divisors by the moduli map $\tilde{H} \rightarrow \overline{\mathcal{M}}_{g}$, both for even or odd genus $g$.

In Sect. 5, we follow $[9,14]$ to establish in Theorem 25 that the completed Hurwitz space $\overline{\mathcal{H}}_{d, g}$, and thus also the normalised level set $\tilde{H}$ for odd $g$, is smooth at a general point of the divisors found in Sect. 4. We discuss the proof with detail, in order to find local models for the divisor formed by the ramification points of the covers (the ramification locus $R$ ) in disks intersecting transversely the divisors in the list.

Theorem 25 concludes our general discussion on how to turn a Lefschetz fibration with fibers of any genus $g$ into a family of ramified covers of the Riemann sphere, thus a finite cover $\kappa: \tilde{X} \rightarrow P$ where $P$ is a symplectic ruled surface. The last section of this work is devoted to discussing in greater detail the case of Lefschetz fibrations of genus $g=4$. This is actually the simplest case beyond the work in genus 2 by Auroux, Siebert and Tian $[2,15,16]$, because the case $g=3$ requires working with a divisor $\tilde{H}$ rather than the entire Hurwitz space, and dealing with the hyperelliptic divisor in moduli space, doubly burdening the subsequent discussion.

In contrast, the case of Lefschetz fibrations with fibers of even genus $g=4$ is simple enough to derive from the fibration and its pullback to Hurwitz space topological information about the original symplectic manifold $X$. Now the Hurwitz space is $\overline{\mathcal{H}}_{3,4}$, and the branched cover $\overline{\mathcal{H}}_{3,4} \rightarrow \overline{\mathcal{M}}_{4}$ has degree 2 . This means that a generic curve of genus 4 admits two trigonal structures, 3:1 covers $C_{4} \rightarrow \mathbb{P}_{\mathbb{C}}^{1}$ with 12 simple ramification points. These trigonal structures are determined by the canonical embedding of the curve, and may be effectively studied in holomorphic families over a disk or a general 1-dimensional base [5].

In Sect. 6 we apply the previous sections to show that for such a symplectic Lefschetz fibration of genus $4 f: X \rightarrow B$ there exists a compatible almost complex structure $J$ and 
a 2:1 pullback family $\tilde{f}: \tilde{X} \rightarrow \tilde{B}$ such that $\tilde{B}$ is a closed Riemann surface, $\tilde{X} \rightarrow X$ a $2: 1$ cover ramified exactly at finitely many regular fibers, and $\tilde{f}$ admits a factorization

$$
\tilde{X} \stackrel{\tilde{\kappa}}{\longrightarrow} P \stackrel{p}{\longrightarrow} \tilde{B}
$$

where $p: P \rightarrow \tilde{B}$ is a family of 2-spheres over $\tilde{B}$ (Proposition 27), such that it admits a compatible symplectic structure making the ramification locus $R \subset P$ of $\kappa$ a symplectic submanifold in its smooth part (Proposition 30), and with local models for $R$ around its singular points listed in Corollary 28. These local models are singularities in complex algebraic curves.

Unfortunately, the last local model in the list corresponds to smooth curves of genus 4 which become singular trigonal coverings of the Riemann sphere, and it can be realized with any orientation (as pointed out in the remark after Theorem 15). This possibility of a conjugate complex orientation in one of the models complicates the study of symplectic fibrations as an extension of the holomorphic ones in genus 4.

\section{Compatible symplectic structures in a 4-dimensional Lefschetz fibration}

Now we will address the question of the uniqueness up to deformation of the symplectic form on a 4-dimensional symplectic Lefschetz fibration $f: X^{4} \rightarrow B$, for $B$ any Riemann surface.

We will start pointing out the difference between critical and regular points:

Lemma 1 Let $p \in X^{4}$ be a critical point of a 4-dimensional symplectic Lefschetz fibration $f$, and $V \subset X^{4}$ a neighbourhood with an adapted oriented complex-valued chart $\left(z_{1}, z_{2}\right)=$ $\left(x_{1}+i y_{1}, x_{2}+i y_{2}\right)$ in which $p=(0,0)$ and $f\left(z_{1}, z_{2}\right)=z_{1}^{2}+z_{2}^{2}$.

If a 2 -form $\omega$ in $V$ given by a skew-symmetric matrix $B\left(x_{1}, y_{1}, x_{2}, y_{2}\right)$ restricts to a positive area form in every vertical subspace in $V$, the inequality

$$
\left(\begin{array}{lll}
y_{2}-x_{2}-y_{1} & x_{1}
\end{array}\right) B(0,0)\left(\begin{array}{c}
x_{2} \\
y_{2} \\
-x_{1} \\
-y_{1}
\end{array}\right) \geq 0
$$

must hold for any $\left(x_{1}, y_{1}, x_{2}, y_{2}\right) \in \mathbb{R}^{4}$.

Proof An immediate computation shows that the tangent space to the fiber of $f$ at any point $\left(x_{1}, y_{1}, x_{2}, y_{2}\right) \in V \backslash\{p\}$ has a positive basis formed by the vectors $\left(y_{2},-x_{2},-y_{1}, x_{1}\right),\left(x_{2}\right.$, $\left.y_{2},-x_{1},-y_{1}\right)$. Multiplying the coordinates by $r>0$ and taking the limit as $r \rightarrow 0$ shows our statement.

In order to simplify our discussion, we introduce some notation:

Definition 2 We will say that a 2-form $\omega$ is strictly compatible with $f: X^{4} \rightarrow B$ at a critical point $p$ if the inequality (1) is strict for nonzero $\left(x_{1}, y_{1}, x_{2}, y_{2}\right)$.

A symplectic form $\omega$ is compatible with $f: X^{4} \rightarrow B$ if it restricts to a symplectic form on all tangent planes to a fiber of $f$, either at regular or singular points of $f$.

The computation of the proof of Lemma 1 shows that the standard symplectic structure $\omega_{0}=d x_{1} \wedge d y_{1}+d x_{2} \wedge d y_{2}$ is strictly compatible with the fibration $\left(z_{1}, z_{2}\right) \mapsto z_{1}^{2}+z_{2}^{2}$. Therefore Gompf's symplectic structure as constructed in [1] is strictly compatible at every critical point. A simple property in the opposite direction is 
Lemma 3 If a 2-form $\omega$ is strictly compatible with the 4-dimensional Lefschetz fibration $f: X^{4} \rightarrow B$ at a critical point $p$, it is nondegenerate in a neighbourhood of $p$.

Proof Choose a coordinate chart $V$ around $p$ as in Lemma 1. If $\omega(p)$ is degenerate, a nonzero isotropic $\left(y_{2},-x_{2},-y_{1}, x_{1}\right)$ cannot obey the strict inequality in (1).

We may now discuss the equivalence of compatible forms:

Proposition 4 Let $f: X^{4} \rightarrow B$ be an oriented, 4-dimensional Lefschetzfibration. The space of symplectic forms $\omega$ on $X^{4}$ compatible with $f$ is arc-connected.

Proof Let $\omega_{0}, \omega_{1}$ be two symplectic forms on $X^{4}$ compatible with $f: X^{4} \rightarrow B$. As we have discussed after Definition 2, $X^{4}$ admits a symplectic structure $\omega$ that is compatible with $f$ and strictly compatible at every critical point. By compactness of $X^{4}$, there exist $\varepsilon_{0}, \varepsilon_{1} \in(0, \infty)$ small enough such that the forms $\omega_{0}^{\prime}=\omega_{0}+\varepsilon_{0} \omega, \omega_{1}^{\prime}=\omega_{1}+\varepsilon_{1} \omega$ are nondegenerate in every point of $X^{4}$. They are also closed, compatible with the fibration and strictly compatible at the critical points, and so are the intermediate forms $\omega_{i}+t \omega$ for $0<t \leq \varepsilon_{i}$.

Consider now $\omega_{B}$ the canonical symplectic structure on the base, and the forms

$$
\omega_{K, t}=(1-t) \omega_{0}^{\prime}+t \omega_{1}^{\prime}+K f^{*} \omega_{B} \quad \text { on } X^{4}, \text { with } K \geq 0 \text { and } t \in[0,1] .
$$

The condition of strict compatibility at a critical point $p$, given by strict inequality in (1) for nonzero $\left(x_{1}, y_{1}, x_{2}, y_{2}\right)$, defines a convex set of forms. Together with the fact that $f^{*} \omega_{B}(p)=$ 0 , this shows that the forms $\omega_{K, t}$ are strictly compatible with the fibration at any critical point $p$ and for every $K \geq 0$ and $t \in[0,1]$.

By Lemma 3 they are also nondegenerate on a neighbourhood of the critical points; they are obviously closed and compatible at regular points.

Let now $V_{1}, \ldots, V_{n}$ be open neighbourhoods of the critical points $p_{1}, \ldots, p_{n}$ such that $\omega_{0, t}$ is nondegenerate in them for every $t \in[0,1]$. The values of the forms $\omega_{K, t}$ do not vary with $K$ on the vertical tangent spaces $T_{p} X_{p}$, whereas in the orthogonal subspaces $\left(T_{p} X_{p}\right)^{\omega_{K, t}}=\left(T_{p} X_{p}\right)^{\omega_{0, t}}$ the term $K f^{*} \omega_{B}$ becomes dominant. Therefore by choosing $K$ large enough, $\omega_{K, t}$ becomes nondegenerate in the compact set $X \backslash \cup_{i=1}^{n} V_{i}$ and for every $t \in[0,1]$. By our assumption of compatibility of orientations, the forms $f^{*} \omega_{B}$ and $\omega_{0, t}$ induce area forms of the same orientation at $\left(T_{p} X_{p}\right)^{\omega_{K, t}}=\left(T_{p} X_{p}\right)^{\omega_{0, t}}$ for every regular point $p \in V_{i}$, so $\omega_{K, t}$ is also nondegenerate at these points for our chosen $K$. As $\omega_{K, t}(p)=\omega_{0, t}(p)$ at every critical point $p$, our claim follows.

\section{Almost complex structures}

2.1 Transversality properties of compatible almost complex structures

Let us assume that $(X, \omega)$ is a compact symplectic 4-manifold and let $f: X \rightarrow B$ be a symplectic Lefschetz fibration on $X$ of genus $g$ with $B$ a closed orientable surface.

Definition 5 An almost complex structure on $X$ is a vector bundle endomorphism

$$
J: T X \longrightarrow T X \text { such that } J^{2}+\mathrm{Id}=0 .
$$

We say that $J$ is compatible with the fibration $f: X \rightarrow B$ if it is compatible with the symplectic form $\omega$, i.e. the tensor

$$
g(u, v)=\omega(u, J v)
$$


is a Riemannian metric on $X$, and that the fibres of $f$ are holomorphic with respect to $J$. This last condition sould be understood in the following manner for the critical points of the singular fibres: for each singular fibre contaning a critical point $p_{i}$ there are two local branches meeting at $p_{i}$; these two branches must be holomorphic with respect to $J$.

The question about the existence of an almost complex structure compatible with the fibration may be interpreted in terms of the structure group of the real tangent bundle $T X \rightarrow$ $X$. At regular points, the fibration structure restricts the structure group from $\operatorname{GL}(4, \mathbb{R})$ to $\operatorname{GL}(2, \mathbb{R}) \times \operatorname{GL}(2, \mathbb{R})$. In turn the symplectic structure on $X$ compatible with the fibration restricts the group to $\operatorname{Sp}(2, \mathbb{R}) \times \mathrm{Sp}(2, \mathbb{R})$, which has compact part $\mathrm{U}(1) \times \mathrm{U}(1)$. This last retraction corresponds to the existence of an almost complex structure $J$ compatible with the fibration and $\omega$; at the same time such a $J$ furnishes $X$ with a hermitian metric on $T X \rightarrow X$ with real part a Riemannian metric and with imaginary part $-2 \omega$.

Recall from [12] further basic definitions:

Definition 6 Let $J$ be an almost complex structure on a symplectic manifold $(X, \omega)$.

- $\quad J$ is $\omega$-tame if, for every $x \in X$ and nonzero $v \in T_{X} X$ one has

$$
\omega(v, J v)>0
$$

- $J$ is $\omega$-compatible if it is tame and, moreover, for every $x \in X$ and $u, v \in T_{x} X$ one has

$$
\omega(J u, J v)=\omega(u, v)
$$

Now we will discuss the uniqueness up to deformation of compatible almost complex structures. First we need an elementary result from symplectic linear algebra.

Lemma 7 Set $E=\mathbb{C}^{n} \cong \mathbb{R}^{2 n}$, let $\omega$ be the standard symplectic form on $E$ and $J_{0}$ the standard complex structure on E. Also let $V \subseteq E$ be a subspace invariant by $J_{0}$ which is symplectic with respect to $\omega$. Consider the following conditions on a complex structure $J$ on $E$,

(1) $J$ is $\omega$-tame.

(2) $J$ is $\omega$-compatible.

(3) $J$ is $\omega$-tame and $V$ is invariant by $J$.

(4) $J$ is $\omega$-tame and $J$ equals some complex structure $J_{V}$ on $V$ (which is $\omega$-tame or $\omega$ compatible).

Then the space of all complex structures $J$ on E satisfying any set of the former conditions is a non empty contractible topological space. Moreover, the spaces of $\omega$-tame complex structures, respectively fulfilling condition (iii) or (iv), retracts strongly to the subspace of those $\omega$-compatible, respectively fulfilling condition (iii) or (iv).

Proof The statements for conditions (i) and (ii) are well known. We follow Sévennec's proof of proposition 2.51 of [13] and add conditions (iii) and $* i v$ ). In a suitable basis of $E$ the matrix of $J_{0}$ is

$$
\left(\begin{array}{cc}
0 & -\mathrm{Id} \\
\mathrm{Id} & 0
\end{array}\right)
$$

where each of the four entries are $n \times n$ sized matrices. The matrix of $\omega$ in the same basis is $-J_{0}$. Note that the standard metric on $\mathbb{R}^{2 n}$ is given by $\langle u, v\rangle=\omega\left(u, J_{0} v\right)$. We will think of complex structures $J$ on $E$ in terms of the tensor $g_{J}(u, v)=\omega(u, J v)$. In coordinates, the matrix of $g_{J}$ is $Z=-J_{0} J$. So there is a bijective corresponce between matrices $J$ satisfying $J^{2}=-$ Id and real matrices $Z$ with $Z^{-1}=-J_{0} Z J_{0}$. Moreover, 
- $J$ is $\omega$-tame $\Leftrightarrow Z>0$, i.e. $\langle u, Z u\rangle>0$ if $u \neq 0$.

- $\quad J$ is $\omega$-compatible $\Leftrightarrow Z>0$ and $Z^{t}=Z$.

- $\quad V$ is invariant by $J \Leftrightarrow V$ is invariant by $Z$.

- $J$ equals $J_{V}$ on $V \Leftrightarrow Z$ equals $\left(-J_{0}\right) J_{V}$ on $V$.

Let us perform the matrix transformation

$$
Z \longmapsto W=(1-Z)(1+Z)^{-1},
$$

which reminds us of the Cayley transformation on the Riemann sphere $w=\frac{1-z}{1+z}$. This transformation is well defined as long as $Z>0$ (i.e. $\langle v, Z v\rangle>0$ for $v \neq 0$ ), in which case $\|W\|<1$ (we use the standard metric $\langle\cdot, \cdot\rangle$ of $E$ ). It is plain to see that $Z^{-1}$ is mapped to $-W$ as long as $Z$ is mapped to $W$. The inverse transformation $W \mapsto Z=(1-W)(1+W)^{-1}$ is well defined as long as $\|W\|<1$. Then,

- $\quad J$ is a complex structure $\Leftrightarrow W=J_{0} W J_{0}$.

- $J$ is $\omega$-tame $\Leftrightarrow\|W\|<1$.

- $J$ is $\omega$-compatible $\Leftrightarrow\|W\|<1$ plus $W^{t}=W$.

- $\quad V$ is invariant by $J \Leftrightarrow V$ is invariant by $W$.

- $\quad J$ equals $J_{V}$ on $V \Leftrightarrow W$ equals $\left(1+J_{0} J_{V}\right)\left(1-J_{0} J_{V}\right)^{-1}$ on $V$.

Now the upshot is that all above conditions on $W$ are convex, hence the space of complex structures satisfying either of them is contractible or empty. Nonemptiness is immediate for the first three conditions, and follows easily by defining $J$ on $V$ and its symplectic orthogonal in the last two.

Finally, the map

$$
W \longmapsto(1-t) W+\frac{t}{2}\left(W+W^{t}\right)
$$

defines a strong deformation retraction from the space of $\omega$-tame complex structures to the subspace of those $\omega$-compatible, which itself retracts to a point by $W \mapsto(1-t) W+t 0$ (note $W=0$ corresponds to $J=J_{0}$ ).

It will convenient for us to study unicity of compatible almost complex structures after choosing local models around singular fibers:

Proposition 8 Let $(f: X \rightarrow B, \omega)$ be a symplectic Lefschetzfibration and $C$ a closed subset of $X$ containing an open neighbourhood of the critical points of $f$. Let $J_{C}$ be a smooth almost complex structure compatible with $f$ and $\omega$ defined over $C$ and let $J^{v}$ be an almost complex structure on the vertical tangent bundle $T X^{v} \rightarrow X$, compatible with the restriction of $\omega$ to the fibres and matching the vertical restriction of $J_{C}$ on $C$. Consider the following conditions on an almost complex structure $J$ on $X$ matching $J_{C}$ on $C$ :

(1) $J$ is $\omega$-tame.

(2) $J$ is $\omega$-compatible.

(3) $J$ is $\omega$-tame and the fibres of $f$ are $J$-holomorphic.

(4) $J$ is $\omega$-tame and matches $J^{v}$ on the fibres of $f$.

Then,

(a) The space of all J's matching $J_{C}$ on $C$ and satisfying any set of the above conditions is a nonempty, contractible space.

(b) Moreover, the space of almost complex structures $J$ matching $J_{C}$, taming $\omega$ and compatible with $f$ retracts strongly to the subspace of those compatible with $\omega$. The same holds if we also require the restriction of $J$ to $T X^{v}$ to match $J^{v}$. 
Proof Let $M=X-\left\{p_{1}, \ldots, p_{n}\right\}$, where $p_{1}$ through $p_{n}$ are the critical points of the fibration $f$. Now we are going to construct the space $\mathcal{J}$ of all the almost complex structures compatible with $f: X \rightarrow B$ and satisfying any set of the first three conditions stated before. We will denote by $\partial_{C}$ the subspace of $\mathcal{J}$ of those $J$ 's matching $J_{C}$ over $C$. The fourth condition will be discussed apart from the first three ones.

This space may be realised as the space of smooth sections of a smooth fibre bundle $\pi: \mathcal{J} \rightarrow M$. Namely, for $p \in M$, the fibre $\mathcal{J}_{p}$ consists of the complex structures $J$ in $T_{p} M$ satisfying the corresponding set of conditions. Therefore, there is a bijective correspondence between $\mathcal{J}_{p}$ and the space $F$ of almost complex structures on $E=\mathbb{C}^{n} \cong \mathbb{R}^{2 n}$ satisfying the set of conditions, which is contractible by Lemma 7 .

Moreover, from the proof of Lemma 7 it follows that $F$ is a convex subspace of the vector space $g l(2 n, \mathbb{R})$. We notice that the fibre bundle $\mathcal{J} \rightarrow M$ can be constructed as that associated to the principal bundle with structure group $G=\mathrm{U}(n-1) \times \mathrm{U}(1)$ obtained from the tangent bundle $T X \rightarrow X$. By looking again at the proof of Lemma 7 we conclude that $G$ acts on $F$ by conjugation, $W \mapsto g^{-1} W g$. This action on $F$ provides a construction of the fibre bundle $\mathcal{J} \rightarrow M$.

We readily identify $\mathcal{J}$ with the space of sections of the fibre bundle $\mathcal{J} \rightarrow M$. Also notice that $J_{C}$ is a section of $\mathcal{J} \rightarrow M$ defined over the closed subset $C$ of $M$. As $J_{C}$ is defined over a closed subset of $M$ and $F$ is contractible, it can be extended [10, Theorem 2.7.1] to a global section of $\mathcal{J} \rightarrow M$ and therefore it is an element of $\mathcal{J}$. This proves that $\mathcal{J}$ is non-empty.

In order to show that $\mathcal{\partial}$ is contractible, we use preceeding observations about convexity of $F$ and the action of $G$ on $F$ given by conjugation $W \mapsto g^{-1} W g$. Given some fixed $W \in \mathcal{J}$, the transformation

$$
W^{\prime} \mapsto(1-t) W^{\prime}+t W
$$

is compatible with the $G$-bundle action, thus provides a retraction of $\mathcal{J}$ to its point $W$. On top, if $W$ and $W^{\prime}$ belong to $\partial_{C}$ then the segment $(1-t) W^{\prime}+t W$ consists of almost complex structures also in $\partial_{C}$.

Now we deal with condition (4) about $J$. As it implies condition (3), we conclude that a $J$ which satisfies fourth condition is a section of the fibre bundle $\mathcal{J} \rightarrow M$ with fibre $F$ satisfying the third condition. Moreover this subspace of sections is also convex, for if $W$ and $W^{\prime}$ match along the vertical tangent spaces so does any of the elements of the segment $(1-t) W^{\prime}+t W$, for $t \in[0,1]$. This shows that, as long as property number four is non void, the space of such $J$ 's is contractible.

As for non emptyness, notice that in order to give a (plain, $\omega$-tame, $\omega$-compatible) $J$ matching $J^{v}$ along the fibres it is sufficient to provide a (plain, $\omega$-tame, $\omega$-compatible) almost complex structure on the horizontal distribution $\left(T M^{v}\right)^{\omega}$, i.e. the symplectic orthogonal to the vertical distribution $T M^{v}$. The space of almost complex structures on this horizontal distribution can be again realised as the sections of a fibre bundle $g^{h} \rightarrow M$ with non empty convex fibre, which is again easly seen to be non empty and contractible. Moreover, as $J_{C}$ is compatible with $\omega$ then $J_{C}$ fixes this horizontal distribution; in other words $J_{C}$ restricts to a section $J_{C}^{h}$ of $g^{h} \rightarrow M$ over $C$. Therefore, sections of $g^{h}$ matching $J_{C}^{h}$ over $C$ provide sections of $\mathcal{J} \rightarrow M$ matching $J_{C}$ over $C$ and fulfilling the fourth condition.

Assertion $(b)$ of the proposition follows easily using similar arguments and the retraction of the last paragraph in Lemma 7.

Notation When the closed set $C \subset X$ of Proposition 8 is of the form $C=f^{-1}(A)$, for $A \subset B$ a closed set containing a neighbourhood of every critical value of $f$, we will denote 
almost complex structures defined over $f^{-1}(A)$, compatible with $f$ and $\omega$ as $J_{A}$, instead of $J_{f^{-1}(A)}$.

It is known that a Lefschetz fibration $f: X \rightarrow B$ admits a compatible almost complex structure. We derive from Proposition 8 further properties:

Corollary 9 (1) Let $f: X \rightarrow B$ be a symplectic Lefschetz fibration and let $\left\{s_{1}, \ldots, s_{k}\right\}$ be a set of distinct points of $B$ containing the singular values of $f$. Assume that $B_{i}$ are pairwise disjoint, closed neighbourhoods of $s_{i}$ in $S^{2}$ and let $A=\cup_{i=1}^{k} B_{i}$. Then, the space of all the compatible almost complex structures on $X$ extending some $J_{A}$ prescribed on $f^{-1}(A)$ is a non-empty contractible space.

(2) Let $U_{1}, \ldots, U_{n}$ be pairwise disjoint open neighbourhoods of the critical points $p_{1}, \ldots$, $p_{n}$ corresponding to analytic local models like those in the definition of Lefschetz. fibration. Let $C=\cup_{i=1}^{n} \bar{V}_{i}$, where $V_{i}$ are open neighbourhoods of $p_{i}$ with $\bar{V}_{i} \subset U_{i}$, and $J_{C}$ the integrable structure coming from the holomorphic local models. Then $J_{C}$ extends to a global compatible $J$ and all such extensions are deformation equivalent.

Note that the second part of the previous corollary shows that there always exists an almost complex structure compatible with a Lefschetz fibration $f: X \rightarrow B$.

\subsection{Transversality to the orbifold locus in $\overline{\mathcal{M}}_{g}$}

We will henceforth work with Lefschetz fibrations $f: X \rightarrow B$ with $X$ a 4-dimensional closed symplectic manifold. The set $A \subset B$ will be a finite set of disjoint, closed balls $\left\{B_{i}\right\}$ with smooth boundary, such that each contains one singular value of $f$ at most as an interior point, no singular value in the boundary $\partial B_{i}$, and such that all singular values of $f$ belong to some such ball.

The restrictions of the fibration $f^{-1}\left(B_{i}\right) \rightarrow B_{i}$ are families of closed oriented surfaces, of a fixed genus $g$, possibly with a singular fibre. The definition of an $f$ - and $\omega$-compatible smooth almost complex structure $J_{A}$ on $f^{-1}(A)$ makes all regular fibers compact Riemann surfaces of said genus $g$, turns the singular fibers into stable genus $g$ curves, and defines a smooth moduli map

$$
\Phi_{J_{A}}: A \rightarrow \overline{\mathcal{M}}_{g}
$$

where $\overline{\mathcal{M}}_{g}$ is the Deligne-Mumford moduli space of stable curves.

The map $\Phi_{J_{A}}$ is defined because the Deligne-Mumford moduli space $\overline{\mathcal{M}}_{g}$ is a coarse moduli space for stable curves of genus $g$. It is a complex projective variety, compactification of the moduli space $\mathcal{M}_{g}$ of compact Riemann surfaces of genus $g$, which gets compactified by the addition of a set of divisors $\Delta_{0}, \Delta_{1}, \ldots, \Delta_{\left\lfloor\frac{g}{2}\right\rfloor}$, defined by stable curves with a single ordinary singularity such that the curve is irreducible for $\Delta_{0}$, or has two irreducible components of genera $i, g-i$ for $\Delta_{i}$, with $i>0$.

As a complex projective variety, $\overline{\mathcal{M}}_{g}$ has orbifold singularities, with an orbifold locus of codimension at least 2 . These singularities correspond to genus $g$ curves with automorphisms (with nongeneric automorphisms for $g \leq 2$ ). Reciprocally, the only families of curves with nongeneric automorphisms at which the moduli space $\overline{\mathcal{M}}_{g}$ is generically smooth are the boundary component $\Delta_{1}$ for any genus $g$ (with an automorphism given by involution on the elliptic component) and the family of hyperelliptic curves in genus $g=3$. Thus there is a decomposition $\overline{\mathcal{M}}_{g}=\overline{\mathcal{M}}_{g}^{\#} \cup \operatorname{Orb}\left(\overline{\mathcal{M}}_{g}\right) \cup \Delta_{1} \cup \mathcal{H}$ ell $l_{3}$, with the orbifold locus a subvariety of codimension $\geq 2$ and $\mathcal{H e l l}_{3}$ empty for $g>3$ and a divisor for $g=3$. The Zariski open subset $\overline{\mathcal{M}}_{g}^{\#}$ is defined by curves without (exceptional for $g \leq 2$ ) automorphisms, and is a fine moduli space for such curves, with universal family of curves 


$$
\bar{U}_{g}^{\#} \rightarrow \overline{\mathcal{M}}_{g}^{\#}
$$

The universal family cannot extend over the subvarieties $\operatorname{Orb}\left(\overline{\mathcal{M}}_{g}\right) \cup \Delta_{1} \cup \mathcal{H}$ ell $l_{3}$, but there exist local smooth finite covers of $\overline{\mathcal{M}}_{g}$ on neighbourhoods of any point in these subvarieties with families of curves defined as versal deformations of the curves in the branching locus $\operatorname{Orb}\left(\overline{\mathcal{M}}_{g}\right) \cup \Delta_{1} \cup \mathcal{H}$ ell $l_{3}$, and which descend to the universal family in its complement (see $[4,8]$ for an ample discussion of moduli spaces of curves).

Lemma 10 Let $D$ be a union of codimension $\geq 2$ subvarieties of $\mathcal{M}_{g}$ not met by the moduli map of $\left.J_{A}\right|_{\partial A}$. There is an almost complex structure $J \in \mathcal{J}_{A}$ such that $\Phi_{J}: B \rightarrow \overline{\mathcal{M}}_{g}$ does not meet $D$ outside A. In particular one can avoid the orbifold locus of the coarse moduli space.

Proof Triangulate $B$ so that $A$ is a subcomplex. Let $C$ be a maximal union of closed triangles such that $A$ is part of $C$ and $J_{A}$ can be extended to an almost complex structure $J$ over $C$ compatible with the fibration, with the moduli not meeting $D$ outside $A$. We will show $C=B$.

Otherwise extend $J$ over a further triangle $T$ according to Proposition 8. Pull the fibration back to the universal covering $U$ of $C \cup T$ and choose a trivialization to obtain a map from $U$ to Teichmüller space. By standard theory this map can be perturbed to avoid the preimage of $D$ in Teichmüller space. Furthermore the perturbation can be taken trivial on the preimage of $C$, where the moduli map of $J$ does not meet $D$.

Restrict the perturbed map to any preimage of $T$. This defines a new almost complex structure on $T$, contradicting the maximality of $C$.

Proposition 11 Let $g>3, f: X \rightarrow B$ a Lefschetz fibration of genus $g$, and $A \subset B a$ neighbourhood of the singular values of $f$ as above. Let $\mathcal{B} \subset \overline{\mathcal{M}}_{g}$ be an analytical subvariety such that the map $\Phi_{J_{A}}: A \rightarrow \overline{\mathcal{M}}_{g}$ meets $\mathcal{B}$ transversally.

Then the subset of $\mathcal{J}_{A}$ consisting of almost complex structures $J$ on $X$ such that $\Phi_{J}: B \rightarrow$ $\overline{\mathcal{M}}_{g}$ is transverse to $\mathcal{B}$ contains a nonempty open subspace.

Proof Our starting point is a $J$ such as in the previous lemma, which gives rise to a $\mathrm{C}^{\infty}$ map $\Phi_{J}: B \backslash A \rightarrow \overline{\mathcal{M}}_{g}^{\#}$ (the divisor $\mathcal{H} \mathrm{ell}_{3}$ is empty because $g>3$, and $\Delta_{1}$ is avoided because all singular fibers lie on $A$ ). Now our problem reduces to deform $\Phi_{J}$ to achieve transversality to $\mathcal{B}^{\# \stackrel{\text { def }}{=}} B \cap \overline{\mathcal{M}}_{g}^{\#}$.

For this, let $\mathcal{C}$ denote the topological space of $\mathcal{C}^{\infty}$ maps $\varphi: B \rightarrow \overline{\mathcal{M}}_{g}^{\#}$ such that $\varphi \mid \bar{A}=$ $\Phi_{J} \mid \bar{A}$. Also define $\mathcal{T}$ as the subspace of $\mathcal{C}$ consisting of those maps transverse to the locus $\mathcal{B}^{\#} \subset \overline{\mathcal{M}}_{g}^{\#}$. It is a well known fact that $\mathcal{T}$ is a dense open subspace of $\mathcal{C}$. Indeed $\Phi_{J} \in \mathcal{T}$, so that there exists an open subspace $U$ of $\mathcal{C}$ containing $\Phi_{J}$. For every $u \in U$ we pull-back the universal family over $\overline{\mathcal{M}}_{g}^{\#}$

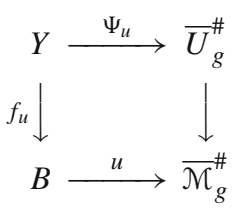

to obtain a Lefschetz fibration $f_{u}: Y \rightarrow S^{2}$ isomorphic to our original fibration $f$, as they have the same monodromy. The locus $\Delta_{1}$ is avoided by small deformations of $u_{\mid B \backslash \AA}$. 
The universal family $\bar{U}_{g}^{\#} \rightarrow \overline{\mathcal{M}}_{g}^{\#}$ carries a Kähler structure $\omega^{\#}$ and an integrable complex structure $J^{\#}$ compatible with $\omega^{\#}$. Note that $J^{\#}$ provides an almost complex structure on $T X^{v} \rightarrow X$, i.e. on the fibres of $f$. However $J^{\#}$ does not provide an almost complex structure on the whole tangent bundle of $X$. Moreover, if $F$ is a fibre of $f$, we cannot assure that $J_{\mid F}^{\#}$ is compatible with $\omega_{\mid F}$.

To overcome this difficulty we will deform $\omega$ to another symplectic form $\omega^{\prime}$ on $X$ compatible with $f$. Namely, fix a symplectic form $\omega_{B}$ on $B$ and call

$$
\omega^{\prime}=\Psi_{u}^{*} \omega^{\#}+K f_{u}^{*} \omega_{B}
$$

which for $K$ large enough is a symplectic form on $X$ compatible with the fibration $f$. Let us see this last assertion more in detail. First, the fibres of $f$ are symplectic with respect to $\omega^{\prime}$. Second, the orientation induced on $X$ coincides with that induced by $\omega$ and the orientation induced by $\omega^{\prime}$ on $B$ is the canonical one provided by $\omega_{B}$. Now Proposition 4 says that $\omega^{\prime}$ is deformation equivalent (through symplectic forms compatible with $f$ ) to $\omega$.

We use existence Proposition 8 to find an almost complex structure $J^{\prime}$ on $X$ compatible with $f$ and $\omega^{\prime}$, matching $J_{A}$ on $A$ and $J^{\#}$ on the fibres of $f$. (This makes sense since $J_{A}$ and $J^{\#}$ are compatible with $\omega^{\prime}$ on $A$ and on the tangent space to the fibres, accordingly.) In summary, we have ended up with a pair $\left(\omega^{\prime}, J^{\prime}\right)$ compatible with the fibration and compatible one each other. Moreover $J^{\prime}$ yields $\Phi_{J^{\prime}}=u$, i.e. we have not modified the sphere in the moduli space. Thus, there is an open subspace of almost complex structures in $\mathcal{J}_{A}$ satisfying the required conditions of the proposition.

Remark If we endow our fibration $f: X \rightarrow B$ with an almost complex structure $J$ as in the previous result, then the sphere $\Phi_{J}$ avoids any undesired closed subvariety of codimension at least 2 plus the orbifold locus Orb $\left(\overline{\mathcal{M}}_{g}\right)$.

\section{Polygonality}

\subsection{Hurwitz spaces of adequate dimension}

The goal of this work is to turn 4-dimensional Lefschetz fibrations, which become families of genus $g$ complex curves once endowed with a suitable almost complex structure, into families of holomorphic covers of the Riemann sphere $\mathbb{P}_{\mathbb{C}}^{1}$ with prescribed degree.

The Hurwitz spaces $\mathcal{H}_{d, g}$ are complex algebraic varieties parametrizing holomorphic morphisms $p: C \rightarrow \mathbb{P}_{\mathbb{C}}^{1}$ of degree $d>0$ and with $C$ any smooth complex projective curve of genus $g$. Each Hurwitz space admits an admissible cover compactification $\overline{\mathcal{H}}_{d, g}$, which is again a complex projective variety, with an orbifold locus, and has a natural projection $\overline{\mathcal{H}}_{d, g} \rightarrow \overline{\mathcal{M}}_{g}$ defined by sending each cover $p$ to its domain $C$, or to its stabilization in the case of singular admissible covers (see $[4,8,14])$.

We want to pull back our Lefschetz fibration $X \rightarrow B$ to the fibre product $\tilde{B}=B \times \overline{\mathcal{M}}_{g} H$ with some Hurwitz space $H$ in order to obtain a polygonal cover structure on the pullback family $\tilde{X} \rightarrow \tilde{B}$. For this purpose we need a Hurwitz space $H$ whose natural projection to moduli space $\overline{\mathcal{M}}_{g}$ is generically finite. For even genus $g$ we may use $H=\overline{\mathcal{H}}_{d, g}$ with $g=2 d-2$. For odd genus $g$, however, no finite projection is available. The projection $\overline{\mathcal{H}}_{d, g} \rightarrow \overline{\mathcal{M}}_{g}$ has generically 1 -dimensional fibers for $d=\frac{g+3}{2}$, so we choose a divisor $H$ in such Hurwitz space $\overline{\mathcal{H}}_{d, g}$ as follows.

For any integer $r \geq 4$, let $\overline{\mathcal{M}}_{0, r}$ be the moduli space of stable curves of genus 0 with $r$ ordered marked points, i.e. the stable completion of the coarse moduli space for objects 
$\left(\mathbb{P}^{1}, p_{1}, \ldots, p_{r}\right.$ ) with $p_{1}, \ldots, p_{r} \in \mathbb{P}^{1}$ distinct points (see [8]). Let $\overline{\mathcal{M}}_{0,[r]}$ be the moduli space of stable curves of genus 0 with $r$ unordered marked points, i.e. the stable completion of the coarse moduli space for objects $\left(\mathbb{P}^{1}, D\right)$ with $D \subset \mathbb{P}^{1}$ a divisor formed by $r$ simple points. For each Hurwitz space $\overline{\mathcal{H}}_{d, g}$, the ramification divisor $D \subset \mathbb{P}^{1}$ of the covers $p: C \rightarrow \mathbb{P}^{1}$ is generically formed by simple branch points and defines a morphism $\overline{\mathcal{H}}_{d, g} \rightarrow \overline{\mathcal{M}}_{0,[r]}$, with $r=2 d+2 g-2$ (see [14]).

We have natural forgetful maps $\pi: \overline{\mathcal{M}}_{0, r} \rightarrow \overline{\mathcal{M}}_{0,[r]}$ given by ignoring the ordering of the points, and $\pi_{I}: \overline{\mathcal{M}}_{0, r} \rightarrow \overline{\mathcal{M}}_{0,4}$ given by any choice of 4 different indices $i_{1}, i_{2}, i_{3}, i_{4} \in$ $\{1, \ldots, r\}$ so that all other $p_{i}$ are ignored. The map $\pi$ is generically finite, with degree $r$ !, while $\overline{\mathcal{M}}_{0,4} \cong \mathbb{P}^{1}$ and each map $\pi_{I}$ is the cross ratio $\left[p_{i_{1}}, p_{i_{2}}, p_{i_{3}}, p_{i_{4}}\right]$.

Let $F$ be a general fibre of one of the cross ratio projections $\pi_{I}$. The image $\bar{F}$ of $F$ in $\overline{\mathcal{M}}_{0,[r]}$ is the locus where one of the cross ratios takes a given value $\varepsilon$. Denote by $V^{\prime}$ the subvariety of Hurwitz space that lies over $\bar{F}$. Choose an irreducible component $V$ such that the image in $\overline{\mathcal{M}}_{g}$ has maximal dimension.

Lemma 12 The projection $V \rightarrow \overline{\mathcal{M}}_{g}$ is dominant and generically finite.

Proof $V^{\prime}$ is not all of Hurwitz space since $\overline{\mathcal{H}}_{d, g} \rightarrow \overline{\mathcal{M}}_{0,[r]}$ is surjective. Thus each irreducible component of $V^{\prime}$ has dimension at most $3 g-3$ and generical finiteness will follow from dominance.

We have to show that $V^{\prime}$ meets a general fibre $F$ of the Hurwitz space over $\overline{\mathcal{M}}_{g}$. The cross ratios of branch points of admissible covers define a subvariety $R$ of $F \times \overline{\mathcal{M}}_{0,4}$. It has positive dimension since it dominates $F$. We have to show that it also dominates $\overline{\mathcal{M}}_{0,4}$.

Otherwise the image of $R$ in $\overline{\mathcal{M}}_{0,4}$ is finite. This means that the cross ratios of any four of the branch points are restricted to finitely many values, which allows only finitely many branch point configurations and, hence, only finitely many admissible coverings. This in turn means that $R$ is finite, a contradiction.

Lemma 13 Stratify Hurwitz space by topological type of admissible coverings and their holomorphic automorphisms, refine the stratification such that it includes the irreducible components and singular loci of all (closed) strata and denote the resulting stratification by $\mathcal{S}$. For general $\varepsilon$ the divisor $V^{\prime}$ does not contain any of the strata.

Proof Assuming the contrary, some cross ratio of branch points is constant $\varepsilon$ on some stratum. This can happen for at most finitely many $\varepsilon$ on each of the finitely many strata.

Corollary 14 If a stratum $S$ of $\mathcal{S}$ meets $V$ then we have $\operatorname{dim}(V \cap S)=\operatorname{dim}(S)-1$ and, hence, the codimension of $V \cap S$ in $V$ is the same as that of $S$ in the full Hurwitz space.

Now, in the odd genus case, we take $H=V$. However, for odd as well as for even genus, we finally have to work with the normalization $\tilde{H}$ of $H$, instead of $H$ itself, because the latter is usually singular in codimension 1 . The Hurwitz stack $\overline{\mathcal{H}}_{d, g}$ has singularities along certain boundary divisors $[14, \S 3.23]$. Furthermore, in the odd genus case our divisor $H=V$ crosses itself in the codimension one locus where two of the cross ratios of branch points take the value $\varepsilon$. Note that $\tilde{H}$ is finite over $H$.

\subsection{Global polygonal structure}

Let $A=\cup B_{i} \subset B$ be a collection of disjoint closed balls with smooth boundary around the critical values of the 4-dimensional Lefschetz fibration $f: X \rightarrow B$, as in Sect. 2.2. We work only with fibrations where the fiber genus is $g>3$ to avoid difficulties with the hyperelliptic 
divisor. We are ready now to find an almost complex structure $J$ with all the properties sought in this work:

Theorem 15 For fiber genus $g>3$, there exists an almost complex structure $J$ on $X$, such that $\tilde{B}=B \times \overline{\mathcal{M}}_{g}$ H is a smooth surface and the pullback fibration $\tilde{X}=X \times \overline{\mathcal{M}}_{g} \tilde{H}$ is a symplectic Lefschetz fibration outside $\AA$ and a stable holomorphic fibration over A.

$\tilde{X}$ admits a map to a ruled surface which is a d-gonal covering with simple branching except for finitely many fibres where, up to perturbing once more the almost complex structure, the local models are:

(1) For even genus $g$ : families over disks transversally intersecting the boundary components of $\overline{\mathcal{H}}_{d, g}$, except in the case of boundary components $D \subset \partial \overline{\mathcal{H}}_{d, g}$ over $\Delta_{1} \subset \partial \overline{\mathcal{M}}_{g}$, where the local model is the family over a disk $U$ transverse to the boundary component $D$ in coarse Hurwitz space $\left|\overline{\mathcal{H}}_{d, g}\right|$, with $U$ mapping 2:1 to a disk transversally intersecting $D$ in $\overline{\mathcal{H}}_{d, g}$.

(2) For odd genus g: families over holomorphic or antiholomorphic disks intersecting the boundary components of $\overline{\mathcal{H}}_{d, g}$ with finite multiplicity.

Proof Endow $\overline{\mathcal{M}}_{g}$ with a stratification including the images of the strata of $\mathcal{S}$ from Lemma 13, the non-finite as well as the non-étale locus of the map $\tilde{H} \rightarrow \overline{\mathcal{M}}_{g}$ from Sect. 3.1 and, as always, the components and singular loci of all (closed) strata.

First of all, choose the complex structure on $\left.X\right|_{A}$ general in the sense that the holomorphic disks $B_{i} \rightarrow \overline{\mathcal{M}}_{g}$ are contained in the open stratum (the part of $\mathcal{M}_{g}^{\#}$ over which all sheets of $\tilde{H}$ are fine moduli spaces and $\tilde{H} \rightarrow \overline{\mathcal{M}}_{g}$ is étale) except for transverse intersections with the boundary of $\overline{\mathcal{M}}_{g}$, in points of $\partial \mathcal{M}_{g}$ that belong to the divisorial strata whose closure is the respective boundary component.

Choose $J$ in the set $\mathcal{J}_{A}$ corresponding to the perturbed disks $A \rightarrow \overline{\mathcal{M}}_{g}$. After Lemma 10 we can arrange that no fibre of $X \rightarrow B$ has non-trivial automorphisms, i. e. over $B \backslash A^{\circ}$ the fibration together with $J$ is given by a differentiable map $\Phi_{J}$ from $B \backslash A^{\circ}$ to $\overline{\mathcal{M}}_{g}^{\#}$, the moduli space of stable genus $g$ curves without special automorphisms. Apply to $\Phi_{J}$ a small generic perturbation fixing the boundary to obtain an almost complex structure on $\left.X\right|_{B \backslash A}$ 。 which fits continuously with the complex structure of $\left.X\right|_{A}$ and is transverse to the aforementioned stratification of $\overline{\mathcal{M}}_{g}$.

Since $\tilde{H}$ is irreducible, the non-finite locus of the projection to $\overline{\mathcal{M}}_{g}$ has at least codimension 2 and is avoided by our perturbed $B \rightarrow \overline{\mathcal{M}}_{g}$. Since $\tilde{H}$ is normal, singularities can occur at most in codimension 2 and do not occur over the image of $B$. Furthermore the branch locus of $\tilde{H} \rightarrow \overline{\mathcal{M}}_{g}$ is met transversely. Thus $\tilde{B}$ is a smooth surface. It even inherits a complex structure from $B$ which makes the projection to $B$ holomorphic. Then the moduli map $\tilde{B} \rightarrow \overline{\mathcal{M}}_{g}$ is holomorphic wherever it hits the boundary. This already implies the first assertion on the pull-back fibration.

Let $\tilde{X} \rightarrow \tilde{B}$ be the pullback fibration defined by $\tilde{B} \rightarrow B$, with its pullback almost complex structure. By the modular property of $\tilde{H}$ the fibration $\tilde{X} \rightarrow \tilde{B}$ factors over a polygonal covering of a ruled surface except for the finitely many special fibres where the moduli map $\tilde{B} \rightarrow \overline{\mathcal{M}}_{g}$ meets a divisorial stratum $D$ in $\overline{\mathcal{M}}_{g}$. Let $\tilde{D}$ be a local sheet of the preimage of $D$ in $\tilde{H}$. By construction, either $\tilde{D}$ is in the open stratum of $\tilde{H}$ or it is a divisorial stratum of $\tilde{H}$. In the first case $D$ is just part of the branch divisor of $\tilde{H} \rightarrow \overline{\mathcal{M}}_{g}$ and the polygonal structure on $\tilde{X}$ extends over the special fibre. So let us assume that $\tilde{D}$ is a divisorial stratum. By Corollary 14 such a stratum comes from a divisorial stratum in the Hurwitz space $\overline{\mathcal{H}}_{d, g}$. This must be 
(an open part of) a boundary component of Hurwitz space, by the transversality properties of $J$ established in Lemma 10.

Observe that locally along $\tilde{D}$ the Hurwitz space $\tilde{H}$ is a cyclic covering of moduli space $\overline{\mathcal{M}}_{g}$ since $D$ is a smooth divisor. Thus if $U \subset B$ is a small disk around a point where $B$ meets $D$ then we have a diagram

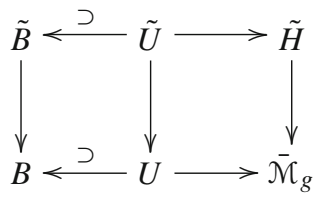

with $\tilde{U}$ a disk covering $U$ with degree $e+1$, totally branched over the center of $U$. Here, $e$ is the ramification order of $\tilde{H} \rightarrow \overline{\mathcal{M}}_{g}$ along $\tilde{D}$.

As a consequence of the smoothness of the completed, coarse Hurwitz space $\left|\overline{\mathcal{H}}_{d, g}\right|$, established in Theorem 25, we may define a fibration $X_{0} \rightarrow U_{0}$ corresponding to a map of a disk $U_{0}$ into the coarse Hurwitz space meeting the boundary in one point of the stratum $\tilde{D}$ and such that the local intersection multiplicity of $U_{0}$ with $\tilde{D}$ is the same as downstairs (for $U$ with $D$ ), i.e. multiplicity 2 if the closure of $D$ is $\Delta_{1}$, and multiplicity 1 (transverse intersection) otherwise. We also show that the universal genus $g$ fibration (though not necessarily the universal admissible covering) exists over $U_{0}$.

For even genus it follows that coarse Hurwitz space $\left|\overline{\mathcal{H}}_{d, g}\right|$ is unramified over coarse moduli space $\left|\overline{\mathcal{M}}_{g}\right|$ along $\tilde{D}$. In the notation from above this means $e=0$ i.e. we can homotop $U$ into $U_{0}$, which corresponds to a deformation of the almost complex structure on $\tilde{X}$ such that in the end $\tilde{X}$ has the required local model. If the special fibre over $U$ is singular then we even know that the orientation of the holomorphic model matches since the monodromy of the symplectic Lefschetz fibration $X \rightarrow B$ along the boundary of $U$ is a positive Dehn twist.

For odd genus, however, we only know the finiteness of $e$. Therefore, we can select families over transverse disks $\tilde{U}$ in the antiimage of $H$ in coarse Hurwitz space (by the transversality properties of $J$ the family avoids the singularities of $H$ ). These transverse disks will project with some finite degree to a disk intersecting the boundary divisors in $\overline{\mathcal{H}}_{d, g}, \overline{\mathcal{M}}_{g}$ with finite degree. As in the even genus case, the orientation of this model will match the symplectic orientation of $\tilde{X}$ around singular fibers of the Lefschetz fibration because of the positivity of the monodromy Dehn twists. But in the case of neibourhoods of regular fibers with a nongeneric $d$-gonal cover the local model may appear with any orientation.

Example 16 To get a Lefschetz fibration of curves where the local model in the $d$-gonal pullback around a regular fiber which is a singular cover has the antiholomorphic orientation, take a smooth sphere $S^{2} \subset \overline{\mathcal{H}}_{d, g}$ intersecting positively the boundary components of Hurwitz space corresponding to covers $C \rightarrow \mathbb{P}_{\mathbb{C}}^{1}$ with $C$ nodal, both positively and negatively some boundary divisor of Hurwitz space corresponding to covers with $C$ smooth, and transversely in all cases.

The family of curves obtained by pullback from the Hurwitz universal family over such inclusion $S^{2} \subset \overline{\mathcal{H}}_{d, g}$ is a chiral Lefschetz fibration, so it admits a symplectic Lefschetz fibration structure by Gompf's theorem (Cor. 10.2.23 in [7]), the local models of Theorem 15 are induced by the defining pullback map of the family, and they appear with both orientations. 


\section{Boundary components of Hurwitz space}

We have to list those boundary components $D$ of Hurwitz space such that $D \cap H$ projects onto a divisor in moduli space $\overline{\mathcal{M}}_{g}$.

\subsection{Reducible fibres}

Here we look for boundary components of Hurwitz space that dominate the divisors $\Delta_{i}$ (with $i \in\left\{1, \ldots,\left\lfloor\frac{g}{2}\right\rfloor\right\}$ of $\overline{\mathcal{M}}_{g}$ parametrizing reducible curves with two components of genera $i, g-i$. As we will look symmetrically at both irreducible components, in this section and next we will follow for convenience the

Notation For $g_{1}, g_{2}$ positive integers with $g_{1}+g_{2}=g$, let $\Delta_{g_{1}, g_{2}}$ be the boundary divisor $\Delta_{\min }\left\{g_{1}, g_{2}\right\}$ of $\partial \overline{\mathcal{M}}_{g}$.

Definition 17 Let $L$ be a nodal curve with two rational components $L_{1}$ and $L_{2}$ meeting in a single point. For $g_{1}, g_{2} \geq 1$ with $g_{1}+g_{2}=g$ and for $v=1, \ldots, d$ define boundary strata of the Hurwitz space as follows.

If $g_{1}, g_{2} \equiv v-1 \bmod 2$ then denote by $\partial_{g_{1} \cup g_{2}, v} \mathcal{H}_{d, g}$ the space of admissible covers of $L$ composed by

(1) a genus $g_{i}$ cover $C_{i}$ of $L_{i}(i=1,2)$ of degree $d_{i}=\left(g_{i}+v+1\right) / 2$, ramified with multiplicity $v-1$ in the unique intersection point $C_{1} \cap C_{2}$

(2) $d-d_{i}$ trivial covers of $L_{i}(i=1,2)$

If $g_{1} \equiv v$ and $g_{2} \equiv v-1 \bmod 2$ then define $\partial_{g_{1} \cup g_{2}, v} \mathcal{H}_{d, g}$ and $\partial_{g_{1} \cup g_{2}, v}^{\prime} \mathcal{H}_{d, g}$ as the stacks of admissible covers according to (1) and (2) but with the following modifications.

- for $\partial_{g_{1} \cup g_{2}, v} \mathcal{H}_{d, g}$ put $d_{1}=\left(g_{1}+v+2\right) / 2$;

- for $\partial_{g_{1} \cup g_{2}, v}^{\prime} \mathcal{H}_{d, g}$, one of the rational components is a double cover of $L_{2}$ and meets $C_{1}$ in a ramified point.

By the proposition of $[14, \S 2.2]$ the boundary divisors $\partial_{g_{1}} \cup g_{2}, \nu \mathcal{H}_{d, g}, \partial_{g_{1} \cup g_{2}, v}^{\prime} \mathcal{H}_{d, g}$ are irreducible.

Proposition 18 A boundary component of Hurwitz space can only dominate $\Delta_{g_{1}, g_{2}}$ if it is the closure of one of the $\partial_{g_{1}} \cup g_{2}, v \mathcal{H}_{d, g}$ or the $\partial_{g_{1} \cup g_{2}, v}^{\prime} \mathcal{H}_{d, g}$ from the above definition.

Proof A boundary divisor is over $\Delta_{g_{1}, g_{2}}$ if it parametrizes admissible coverings $C \rightarrow L$ with two smooth components $C_{i}$ of genus $g_{i}(i=1,2)$ projecting to $L$ with some index $d_{i} \leq d$. Then all further components of $C$ are smooth rational and the dual graph of $C$ is a tree.

Among the $r$ ramification points over the smooth locus of $L$ there are $r_{i}$, say, belonging to $C_{i}$ and $r^{\prime}$ belonging to bubbles with $r_{1}+r_{2}+r^{\prime}=r$. The branch point configuration of $C_{i} \rightarrow L$ may or may not include the node of $L$ and depends, thus, on $r_{1}-3$ resp. $r_{1}-2$ moduli. Since the configuration determines, up to finitely many choices, $C_{i}$ as an abstract curve and in the second case also the points where to glue the other components, for dominating $\Delta_{g_{1}, g_{2}}$ we need at least $3 g_{i}-3$ resp. $3 g_{i}-2$ moduli. In both cases this amounts to $r_{i} \geq 3 g_{i}$. Since we have $r=3 g$ if $g$ is even and $r=3 g+1$ if $g$ is odd, we are left with three cases:

(1) $g$ even, $r_{i}=3 g_{i}, r^{\prime}=0$

(2) $g$ odd, $r_{1}=3 g_{1}, r_{2}=3 g_{2}+1, r^{\prime}=0$

(3) $g$ odd, $r_{i}=3 g_{i}, r^{\prime}=1$ 
The fibre over the node of $L$ consists of at most one point of $C_{1} \cap C_{2}$, counting $v$-fold in the fibre if the ramification order is $v-1$ on both branches, furthermore $d_{i}-v$ points of $C_{i}$ and $d^{\prime}$ points outside $C_{1} \cup C_{2}$; everything counted with multiplicities.

Assume $C_{i}$ has total ramification order $e_{i}$ over the node of $L$. Subtract the RiemannHurwitz formulae $r_{i}+e_{i}=2\left(d_{i}+g_{i}-1\right)$ from $r=2(d+g-1)$ to obtain

$$
r^{\prime}-\left(e_{1}+e_{2}\right)=2\left(d-d_{1}-d_{2}+1\right)=2\left(d^{\prime}-v+1\right)
$$

Since $r^{\prime} \leq 1$ we infer $v \geq 1$, which means that $C_{1}$ and $C_{2}$ meet and the common point has ramification order $v-1$ on both branches. But this ramification is part of $e_{i}$. So in any case (2) implies $d^{\prime}=0$; if $r^{\prime}=0$ then $e_{i}=v-1$ so there cannot be a further ramified node. In case $r^{\prime}=1$ we have, apart from $C_{1} \cap C_{2}$, one simply ramified node on either $C_{1}$ or $C_{2}$. Of course it must be on the component $C_{i}$ with $r_{i}+v-1$ odd, since the total ramification order of $C_{i} \rightarrow L_{i}$ must be even.

Remark In the odd genus case we are properly interested not in boundary strata dominating $\Delta_{g_{1}, g_{2}}$ but such that their intersection with $\tilde{H}$ dominates $\Delta_{g_{1}, g_{2}}$. However, when intersecting with $\tilde{H}$ we do not lose dominance. For example, that $\partial_{g_{1} \cup g_{2}, v}^{\prime} \mathcal{H}_{d, g} \cap \tilde{H}$ actually dominates the boundary divisor $\Delta_{g_{1}, g_{2}}$ of $\overline{\mathcal{M}}_{g}$ is due to the observation that given any admissible cover of the type described by $\partial_{g_{1}}^{\prime} \cup g_{2}, v \mathcal{H}_{d, g}$ we can deform the projection to $L$ on the two-sheeted bubble only; in this way we can adjust a cross ratio of branch points involving the ramification of the bubble to become $\varepsilon$ without changing the stable genus $g$ curve.

\subsection{Irreducible singular fibres}

Next we look for boundary components of Hurwitz space that dominate the divisor $\Delta_{0}$ of $\overline{\mathcal{M}}_{g}$ parametrizing irreducible singular curves. The argument is similar to the reducible case but simpler.

Definition 19 Again let $L$ be a nodal curve with two rational components $L_{1}$ and $L_{2}$ meeting in a single point. Define a boundary stratum $\partial_{i r r} \mathcal{H}_{d, g}$ of the Hurwitz space as the space of admissible covers of $L$ composed by

- a $d$-fold genus $g-1$ cover $C_{1} \rightarrow L_{1}$

- $d-2$ trivial and 1 rational double cover of $L_{2}$, meeting $C_{1}$ in unramified nodes

Again by the proposition of $[14, \S 2.2]$ the boundary divisor $\bar{\Delta}_{0}^{\prime}$ is irreducible.

Proposition 20 The closure of $\partial_{i r r} \mathcal{H}_{d, g}$ is the only boundary component of Hurwitz space $\overline{\mathcal{H}}_{d, g}$ that dominates $\Delta_{0}$ in $\overline{\mathcal{M}}_{g}$.

Proof A boundary divisor is over $\Delta_{0}$ if it parametrizes admissible coverings $C \rightarrow L$ with a smooth component $C_{1}$ of genus $g-1$ such that two of its points over the node are connected by an unstable chain of rational components of $C$. All components of $C$ except $C_{1}$ must be smooth rational. The dual graph of $C$ has one cycle.

The number $r_{1}$ of ramification points of $C_{1}$ over $L_{1} \backslash L_{2}$ must satisfy $r_{1}+e_{1}=2\left(g+d_{1}-2\right)$, where $d_{1}$ is the degree of the map $C_{1} \rightarrow L_{1}$ and $e_{1}$ is the total ramification number of $C_{1}$ over the node of $L$. On the other hand the curve $C_{1}$ together with the two points to be identified in the stable model of $C$ depends on $r_{1}-2$ moduli (the branch points on $L_{1} \backslash L_{2}$ and the node of $L$ ). In order to dominate $\mathcal{M}_{g-1,[2]}$ we need at least $3 g-4$ moduli.

This amounts to $r_{1} \geq 3 g-2$ and then $d_{1} \geq(g+2) / 2$. Since $d$ is either $(g+2) / 2$ or $(g+3) / 2$ we conclude $d_{1}=d$ i.e. $C_{1}$ is the only component over $L_{1}$. Then over $L_{2}$ we must have one 
double cover and $d-2$ trivial covers in order that $g$ is the total arithmetic genus. Consequently $L_{2} \backslash L_{1}$ contains $r_{2}=2$ branch points. Use $r_{1}+e_{1}=2\left(g+d_{1}-2\right)=2(g+d-2)=r-2$ and $r_{1}+r_{2}=r$ to conclude $e_{1}=0$, all nodes are unramified.

\subsection{Regular fibres}

Definition 21 Again let $L$ be a nodal curve with two rational components $L_{1}$ and $L_{2}$ meeting in a single point. Define a boundary stratum $\partial_{e=1+1} \mathcal{H}_{d, g}$ of the Hurwitz space as the space of admissible covers composed by

- a $d$-fold cover of $L_{1}$, of genus $g$;

- $d-4$ trivial and 2 rational double covers of $L_{2}$, the double covers meeting the genus $g$ component in simply ramified nodes.

Define $\partial_{e=2} \mathcal{H}_{d, g}$ as the space of admissible covers with

- a $d$-fold cover $C_{1}$ of $L_{1}$, of genus $g$;

- a rational triple cover $C_{2}$ and $d-3$ trivial covers $C_{3}, \ldots, C_{d-1}$ of $L_{2}$; the triple cover meeting $C_{1}$ in a doubly ramified node.

If $g$ is odd then define additionally boundary strata as follows. Let $\partial_{e=1+1+1} \mathcal{H}_{d, g}$ be the space of admissible covers with

- a $d$-fold genus $g$ cover of $L_{1}$

- $d-6$ trivial and 3 rational double covers of $L_{2}$; the double covers meeting the genus $g$ component in simply ramified nodes.

Let $\partial_{e=2+1} \mathcal{H}_{d, g}$ be the space of admissible covers with

- $\quad$ a $d$-fold genus $g$ cover of $L_{1}$

- $d-5$ trivial, 1 double and 1 triple rational cover of $L_{2}$; the double resp. triple cover meeting the genus $g$ component in simply resp. doubly ramified nodes.

Let $\partial_{e=3} \mathcal{H}_{d, g}$ be the space of admissible covers with

- $\quad$ a $d$-fold genus $g$ cover of $L_{1}$

- $\quad d-4$ trivial and 1 rational quadruple cover of $L_{2}$; the quadruple cover meeting the genus $g$ component in a triply ramified node.

Let $\partial_{e=1} \mathcal{H}_{d, g}$ be the space of admissible covers with

- a $(d-1)$-fold genus $g$ cover and a trivial cover of $L_{1}$

- $d-3$ trivial covers and a rational triple cover of $L_{2}$; the triple cover meeting the genus $g$ component in a simply ramified node and the trivial cover of $L_{1}$ in an unramified node.

Let $\partial_{e=1}^{\prime} \mathcal{H}_{d, g}$ be the space of admissible covers with

- a $(d-1)$-fold genus $g$ cover and a trivial cover of $L_{1}$

- $d-4$ trivial covers and 2 rational double covers of $L_{2}$; the first double cover meeting the genus $g$ component in a simply ramified node; the second double cover meeting both components over $L_{1}$ in unramified nodes.

Proposition 22 The closures of the boundary strata defined above are the only boundary divisors of Hurwitz space dominating a divisor in $\mathcal{M}_{g}$. 
Proof If a boundary component of Hurwitz space dominates a divisor in $\mathcal{M}_{g}$ then it generically parametrizes admissible covers $C \rightarrow L_{1} \cup L_{2}$ with one component $C_{1} \subset C$ smooth of genus $g$. The other components of $C$ are smooth rational and the intersection graph is a tree. Assume that $C_{1}$ covers $L_{1}$ with $d_{1}$ sheets. Assume further that $e_{1}$ ramification points (counting with multiplicity) are over the node of the base. Then we have $2\left(d_{1}+g-1\right)-e_{1}$ further ramification points in $C_{1}$, so the number of moduli for $C_{1}$ is bounded by $2 d_{1}+2 g-4-\min \left\{1, e_{1}\right\}$.

On the other hand this number must be at least $3 g-4$ in order to dominate a divisor in $\mathcal{M}_{g}$. We conclude $g+\min \left\{1, e_{1}\right\} \leq 2 d_{1}$. But clearly $d_{1}$ is at most $d$, which is $(g+2) / 2$ for even $g$ and $(g+3) / 2$ else. Thus $d_{1}$ is either $d$ or $d-1$; for the latter case $g$ must be odd.

Assume first that $g$ is even. Then $d_{1}=d$ (so $C_{1}$ is the only component over $L_{1}$ ) and $e_{1} \leq 2$. Since $C_{1}$ has $r_{1}=r-e_{1}$ ramification points besides the nodes and at least 2 branch points must be left for $L_{2}$ to be stable, the only possibility is $e_{1}=2$.

If $e_{1}=2$ comes from a double ramification point then the admissible covering has a three-sheeted component over $L_{2}$ and $d-3$ trivial ones. Otherwise $e_{1}=2$ comes from two simple ramification points with the same image in $L_{1}$ and the admissible covering consists of two double covers and $d-4$ trivial covers of $L_{2}$.

Consider now the odd genus case. Again $L_{2}$ needs at least two branch points to be stable. Recall that we assumed that the boundary divisor $D$ dominates a divisor in $\mathcal{M}_{g}$. If $L_{2}$ has three (or more) branch points then this implies that $D \cap \tilde{H}$ dominates the same divisor in $\mathcal{M}_{g}$, since we may adjust the branch points on $L_{2}$ with respect to the node such that the cross ratio be $\varepsilon$. Otherwise, i.e. if $L_{2}$ has only two branch points then the covering $C \rightarrow L_{1}$ needs $3 g-3$ moduli in order that $D \cap \tilde{H}$ dominates a divisor in $\mathcal{M}_{g}$.

Thus in the subcase with two branch points on $L_{2}$ we conclude $2 d_{1} \geq g+\min \left\{1, e_{1}\right\}+1$. Then $d_{1}=d=(g+3) / 2$ and $e_{1}=2$. This leads to the two configurations we have already seen in the even genus case.

Now consider the subcase with (at least) three smooth branch points on $L_{2}$ and $d_{1}=d$. Then, since $e_{1} \leq 3$, the number of smooth branch points on $L_{2}$ must be exactly three and $e_{1}=3$ may come from

(a) three simple ramification points, or

(b) a double and a simple ramification point, or

(c) a triple ramification point

on $C_{1}$ such that their images on $L_{1}$ coincide.

Finally consider the subcase with (at least) three smooth branch points on $L_{2}$ and $d_{1}=$ $d-1$. Then $e_{1} \leq 1$. For three branch points on $L_{2}$ we actually need $e_{1}=1$. Over $L_{1}$ we have $C_{1}$ and a trivial cover. In order to connect them we need either a three-sheeted component over $L_{2}$, meeting $C_{1}$ in the ramified node and exhibiting the three branch points on $L_{2}$, or a double cover of $L_{2}$ meeting $C_{1}$ in an unramified node, and a further double cover of $L_{2}$ meeting $C_{1}$ in the ramified node.

Remark Note that the above discussion includes the divisor of hyperelliptic genus 3 curves. For $d=3$ the only boundary divisors of Hurwitz space dominating divisors in the interior of moduli space are the two boundary components with $d_{1}=3$ and $e_{1}=2,3$, and the two boundary components with $d_{1}=2$ and $e_{1}=1$. In the genus 3 case the latter two components cover the hyperelliptic locus.

\section{Smoothness of the Hurwitz space}

We appeal to $[14, \S 2.2]$ for the 
Theorem 23 (Hurwitz et al., Mochizuki) The boundary strata introduced above in the non-normalized Hurwitz space, to wit $\partial_{g_{1} \cup g_{2}, v} \mathcal{H}_{d, g}, \partial_{g_{1} \cup g_{2}, v}^{\prime} \mathcal{H}_{d, g}, \partial_{i} r r \mathcal{H}_{d, g}, \partial_{e=1+1} \mathcal{H}_{d, g}$, $\partial_{e=2} \mathcal{H}_{d, g}, \partial_{e=1+1+1} \mathcal{H}_{d, g}, \partial_{e=2+1} \mathcal{H}_{d, g}, \partial_{e=3} \mathcal{H}_{d, g}, \partial_{e=1} \mathcal{H}_{d, g}$, and $\partial_{e=1}^{\prime} \mathcal{H}_{d, g}$, are irreducible.

Corollary 24 The irreducible boundary components of the normalized coarse Hurwitz space dominating divisors in $\overline{\mathcal{M}}_{g}$ correspond bijectively to the topological types of singular fibres, i. e. the boundary strata from the previous theorems.

This follows from Theorem 23 and the following.

Theorem 25 Coarse Hurwitz space $\left|\overline{\mathcal{H}}_{d, g}\right|$ is smooth at a general point of any of the aforementioned divisors.

Proof We follow [9, p. 62] for the local geometry of Hurwitz space near a boundary divisor; see also $[14, \S 3.23]$. Assume the boundary divisor parametrizes admissible coverings $C \rightarrow$ $L$ of $L=L_{1} \cup L_{2}$ as above with smooth branch points $b_{1}, \ldots, b_{r} \in L$. Consider the germ of $\overline{\mathcal{M}}_{0, r}$ at $L$, i.e. a formal universal deformation $L_{M} \rightarrow M=\operatorname{Spec}\left(\mathfrak{o}_{M}\right)$ of the pointed curve $L$. Here $\mathfrak{o}_{M}=\mathbb{C} \llbracket t_{1}, \ldots, t_{r-3} \rrbracket$ is a power series ring with some cross ratios $t_{2}, \ldots, t_{r-3}$ determining the position of the marked points $b_{1}, \ldots, b_{r}$ whereas $t_{1}$ parametrizes the deformation of the node, i.e. the universal stable curve $L_{M} \rightarrow M$ is given by $\mathfrak{o}_{M} \rightarrow$ $\mathfrak{o}_{M}[u, v] /\left(u v-t_{1}\right)$ near the node of the central fibre. Here $u$ is a local coordinate on $L_{1}$ and $v$ a local coordinate on $L_{2}$.

Enumerate the preimages $N_{1}, N_{2}, \ldots \in C$ of the node $L_{1} \cap L_{2}$ and denote by $e_{i}(i=$ $1,2, \ldots$ ) the ramification index of both branches of $C$ at $N_{i}$. The pull-back of the deformation $L_{M}$ to a germ of the Hurwitz stack

$$
H=\operatorname{Spec}\left(\mathfrak{o}_{H}\right), \quad \mathfrak{o}_{H}=\mathfrak{o}_{M}\left[s_{1}, s_{2}, \ldots\right] /\left(s_{1}^{e_{1}}-t_{1}, s_{2}^{e_{2}}-t_{1}, \ldots\right),
$$

admits an admissible covering $C_{H} \rightarrow L_{H}$ deforming $C \rightarrow L$. Note that $H$ is singular if $C \rightarrow L$ ramifies at more than one of the nodes $N_{1}, N_{2}, \ldots$ The complete local ring of $C_{H}$ near $N_{i}$ is $\mathfrak{o}_{H}\left[x_{i}, y_{i}\right] /\left(x_{i} y_{i}-s_{i}\right)$ with the projection to $L_{H}$ given by $u=x_{i}^{e_{i}}$ and $v=y_{i}^{e_{i}}$.

We will have to determine the group $G$ of automorphisms of the admissible covering $C \rightarrow L$, since the germ of coarse Hurwitz space at the moduli point of $C \rightarrow L$ is the quotient of $H$ by this group. More precisely we will consider the group $G$ of automorphisms acting trivially on the stable reduction of $C$, and the quotient $|H|=H / G$. Note that $G$ is the full automorphism group of $(C \rightarrow L)$ except for two special cases, namely hyperelliptic curves of genus 3 or reducible curves with a genus 1 component. In these cases $G$ has index 2 and $|H|=H / G$ will be a local double cover of the coarse Hurwitz space; otherwise $|H|$ is just the germ of $\left|\overline{\mathcal{H}}_{d, g}\right|$. In algebraic terms, the local ring $\mathfrak{o}_{|H|}$ of $|H|$ is the invariant ring $\left(\mathfrak{o}_{H}\right)^{G}$.

Now $C \rightarrow L$ cannot admit any covering transformations other than involutions of twosheeted components, because every multi-sheeted component contains a simple ramification point. However, in contrast to [9] we work with unlabelled branch points. Thus $G$ also involves automorphisms of $L$ that lift to $C$.

In each case $\mathfrak{o}_{|H|}$ will have the form $\mathbb{C} \llbracket w, t_{2}, \ldots, t_{r-3} \rrbracket$. Thus setting $t_{2}=\cdots=t_{r-3}=0$ we obtain a curve germ $W$ transverse to the boundary, resp. meeting the boundary with order 2 in the special cases of hyperelliptic genus 3 curves and reducible curves with elliptic components. The universal admissible cover pulled back to the punctured germ $W \backslash \partial \mathcal{H}_{d, g}$ specializes to a fibre $C / G$ over the boundary point.

In some cases we will blow down $L_{2}$ to obtain a $\mathbb{P}^{1}$-bundle $\bar{L}_{M} \rightarrow M$. This pulls back to a $\mathbb{P}^{1}$-bundle $\bar{L}_{H} \rightarrow H$ and then descends to $\mathbb{P}^{1}$-bundles over $|H|$ and $W$ provided that $G$ acts 
trivially on $L_{1}$. Similarly, within $C_{H}$, we can blow down components covering $L_{2}$ trivially. For components covering $L_{2}$ non-trivially we will have to check in each case if they can be blown down to a smooth point in $C_{H} \rightarrow H$.

How does the branch locus in $\bar{L}_{M}$ look like? The universal sections $b_{1}, \ldots, b_{r_{2}}$ of $L_{M} \rightarrow$ $M$ passing through $L_{2}$ may be given by $v=\zeta_{i}+O\left(t_{2}, \ldots, t_{r-3}\right)\left(i=1, \ldots, r_{2}\right)$ where $\zeta_{i}$ are the $r_{2}$-th roots of unity. Thus in $\bar{L}_{M}$ the equation is $u^{r_{2}}=t_{1}^{r_{2}}+O\left(t_{2}, \ldots, t_{r-3}\right)$ and over $W$ it is $u^{r_{2}}=t_{1}^{r_{2}}$ where $t_{1}$ has to be expressed in $w$ (recall that $w$ is the coordinate on $W$ ).

Let us make this explicit for each individual boundary divisor.

The boundary divisor $\partial_{g_{1} \cup g_{2}, v} \mathcal{H}_{d, g}$. We have a ramified node $N_{1}$ and trivial $G$, thus $\mathfrak{o}_{|H|}=\mathfrak{o}_{H}=\mathbb{C} \llbracket s_{1}, t_{2}, \ldots, t_{r-3} \rrbracket$ with $s_{1}^{v}=t_{1}$. Take $w=s_{1}$. The singularity of the branch divisor in $\bar{L}_{W}$ is given by $u^{r_{2}}=w^{r_{2} v}$.

The local ring of $C_{W}$ at the ramified node, $\mathfrak{o}_{W}\left[x_{1}, y_{1}\right] /\left(x_{1} y_{1}-w\right)$, is regular. However at the unramified nodes the local ring $\mathfrak{o}_{W}\left[x_{i}, y_{i}\right] /\left(x_{i} y_{i}-w^{v}\right)$ is singular unless $v=1$. Since the unstable components are trivial covers of $L_{1}$ resp. $L_{2}$ we can blow down all of them and get a smooth total space $\bar{C}_{W} \rightarrow W$. (Then there is no polygonal map any more.) This also shows that the monodromy is a simple Dehn twist. In the even genus case this means that $\left|\overline{\mathcal{H}}_{d, g}\right|$ does not ramify over $\overline{\mathcal{M}}_{g}$ along $\partial_{g_{1} \cup g_{2}, \nu} \mathcal{H}_{d, g}$.

The boundary divisor $\partial_{g_{1} \cup g_{2}, v}^{\prime} \mathcal{H}_{d, g}$. We have a doubly ramified node $N_{2}$ and a simply ramified node $N_{1}$, thus $\mathfrak{o}_{H}=\mathfrak{o}_{M}\left[s_{1}, s_{2}\right] /\left(s_{1}^{3}-t_{1}, s_{2}^{2}-t_{1}\right)$. On the other hand the involution of the two-sheeted component over $L_{2}$ sends $y_{2}$ to $-y_{2}$, so the invariant ring is $\mathfrak{o}_{|H|}=$ $\mathfrak{o}_{M}\left[s_{1}\right] /\left(s_{1}^{3}-t_{1}\right)=\mathbb{C} \llbracket s_{1}, t_{2}, \ldots, t_{r-3} \rrbracket$. Take $w=s_{1}$. Again the singularity of the branch divisor in $\bar{L}_{W}$ is given by $u^{r_{2}}=w^{r_{2} v}$.

The discussion of the local model proceeds as above but we also have to deal with the simply ramified node, with local ring $\mathfrak{o}_{W}\left[x_{2}, y_{2}\right] /\left(x_{2} y_{2}-w^{v / 2}\right)$ if $v$ is even and $\mathfrak{o}_{W}\left[x_{2}, y_{2}\right] /$ $\left(\left(x_{2} y_{2}\right)^{2}-w^{v}\right)$ if $v$ is odd.

The boundary divisor $\partial_{i r r} \mathcal{H}_{d, g}$. In this case we want to blow down the unstable components. First blow down $L_{2}$ to obtain a ruled surface $\bar{L}_{W}$. The corresponding blow-down of $C_{W}$ is the fibration $\bar{C}_{W} \rightarrow W$ corresponding to the map $W \rightarrow \mathcal{M}_{g}$.

We have $\mathfrak{o}_{H}=\mathfrak{o}_{M}$ since the nodes of $C$ are unramified. $G$ is generated by an order two automorphism defined on $L_{2}$ by fixing the node and exchanging the branch points of the two-sheeted component. This has order two on every component of $C$ over $L_{2}$ and, hence, must send every $y_{i}$ to $-y_{i}$. Consequently, $\tau^{*}\left(t_{1}\right)=-t_{1}$, and $\mathfrak{o}_{|H|}=\mathbb{C} \llbracket w, t_{2}, \ldots, t_{r-3} \rrbracket$ with $w=t_{1}^{2}$.

The branch divisor $u^{2}=w$ in $\bar{L}_{W}$ is smooth and hence so is $\bar{C}_{W}$. (This also means that the monodromy is a simple Dehn twist, so in the even genus case coarse Hurwitz space is unramified over $\overline{\mathcal{M}}_{g}$ along the divisor of irreducible singular curves.)

Regular stable reduction. Whenever the stable reduction of $C$ is smooth we will again want to blow down the unstable components. This will be possible for the same reason as in the case of $\partial_{i r r} \mathcal{H}_{d, g}$. The blow-down of $C_{W}$ is the smooth fibration $\bar{C}_{W} \rightarrow W$ corresponding to the map $W \rightarrow \mathcal{M}_{g}$. This is clearly smooth.

The boundary divisor $\partial_{e=1+1} \mathcal{H}_{d, g}$. The admissible covering has two ramified nodes $N_{1}, N_{2}$. Thus $\mathfrak{o}_{H}=\mathbb{C} \llbracket s_{1}, s_{2}, t_{2}, \ldots, t_{r-3} \rrbracket /\left(s_{1}^{2}-s_{2}^{2}\right)$. The group $G$ is generated by the involutions $\tau_{1}, \tau_{2}$ of the two-sheeted components, with $\tau_{i}^{*}\left(y_{i}\right)=-y_{i}$ and hence $\tau_{i}^{*}\left(s_{i}\right)=-s_{i}$. The invariant ring is $\mathfrak{o}_{|H|}=\mathfrak{o}_{M}$. Take $w=t_{1}$. The singularity of the branch locus is given by $u^{2}=w^{2}$ in $\bar{L}_{W}$.

The boundary divisor $\partial_{e=2} \mathcal{H}_{d, g}$. The three-sheeted component $C_{2}$ ramifies totally over the node and simply over two further points $b_{1}, b_{2} \in L_{2}$. Thus $G=\langle\tau\rangle$ generated by the involution $\tau$ which acts on $L_{2}$ by fixing the node and exchanging $b_{1}, b_{2}$. Let $N_{1}$ be the 
node $C_{1} \cap C_{2}$. Since $\tau$ has order two on each component over $L_{2}$, it must send each $y_{i}$ to $-y_{i}$. The $x_{i}$ are mapped identically. We have $\mathfrak{o}_{H}=\mathbb{C} \llbracket s_{1}, t_{2}, \ldots, t_{r-3} \rrbracket$ with $s_{1}^{3}=t_{1}$ and $\mathfrak{o}_{|H|}=\mathbb{C} \llbracket w, t_{2}, \ldots, t_{r-3} \rrbracket$ with $w=s_{1}^{2}$. The singularity of the branch locus is given by $u^{2}=t_{1}^{2}$ i.e. $u^{2}=w^{3}$ in $\bar{L}_{W}$.

The boundary divisor $\partial_{e=1+1+1} \mathcal{H}_{d, g}$. The admissible covering has three ramified nodes $N_{1}, N_{2}$. Thus $\mathfrak{o}_{H}=\mathfrak{o}_{M}\left[s_{1}, s_{2}, s_{3}\right] /\left(s_{1}^{2}-t_{1}, s_{2}^{2}-t_{1}, s_{3}^{2}-t_{1}\right)$. The group $G$ is generated by the involutions $\tau_{1}, \tau_{2}, \tau_{3}$ of the two-sheeted components, with $\tau_{i}^{*}\left(y_{i}\right)=-y_{i}$ and hence $\tau_{i}^{*}\left(s_{i}\right)=-s_{i}$. The invariant ring is $\mathfrak{o}_{|H|}=\mathfrak{o}_{M}$. Take $w=t_{1}$. The singularity of the branch divisor is given by $u^{3}=w^{3}$ in $\bar{L}_{W}$.

The boundary divisor $\partial_{e=2+1} \mathcal{H}_{d, g}$. The admissible covering has a simply ramified node $N_{1}$ and a doubly ramified node $N_{2}$. Thus $\mathfrak{o}_{H}=\mathfrak{o}_{M}\left[s_{1}, s_{2}\right] /\left(s_{1}^{2}-t_{1}, s_{2}^{3}-t_{1}\right)$. The group $G$ is generated by the involution $\tau$ of the two-sheeted component of $C$, with $\tau^{*}\left(y_{1}\right)=-y_{1}$ and hence $\tau^{*}\left(s_{1}\right)=-s_{1}$. The invariant ring is $\mathfrak{o}_{|H|}=\mathbb{C} \llbracket s_{2}, t_{2}, \ldots, t_{r-3} \rrbracket$ with $s_{2}^{3}=t_{1}$. Take $w=s_{2}$. The singularity of the branch divisor is given by $u^{3}=w^{9}$ in $\bar{L}_{W}$.

The boundary divisor $\partial_{e=3} \mathcal{H}_{d, g}$. Now we deal with a triply ramified node $N_{1}$ and $G$ is trivial. Thus $\mathfrak{o}_{|H|}=\mathfrak{o}_{H}=\mathbb{C} \llbracket s_{1}, t_{2}, \ldots, t_{r-3} \rrbracket$ with $s_{1}^{4}=t_{1}$. Take $w=s_{1}$. The singularity of the branch divisor is given by $u^{3}=w^{12}$ in $\bar{L}_{W}$.

The boundary divisor $\partial_{e=1} \mathcal{H}_{d, g}$. We have a simply ramified node $N_{1}$ and trivial $G$. Thus $\mathfrak{o}_{|H|}=\mathfrak{o}_{H}=\mathbb{C} \llbracket s_{1}, t_{2}, \ldots, t_{r-3} \rrbracket$ with $s_{1}^{2}=t_{1}$. Take $w=s_{1}$. The singularity of the branch divisor is given by $u^{3}=w^{6}$ in $\bar{L}_{W}$.

The boundary divisor $\partial_{e=1}^{\prime} \mathcal{H}_{d, g}$. We have a simply ramified node $N_{1}$ and $G$ generated by the involution $\tau$ of the two-sheeted component containing $N_{1}$. Thus $\mathfrak{o}_{H}=\mathfrak{o}_{M}\left[s_{1}\right] /\left(s_{1}^{2}-t_{1}\right)$ and $\mathfrak{o}_{|H|}=\mathfrak{o}_{M}$. The singularity of the branch divisor is given by $u^{3}=w^{3}$ in $\bar{L}_{W}$.

We have seen that in all cases $|H|$ is smooth. As explained above, the germ of Hurwitz space is the quotient of $|H|$ by the group of those automorphisms of the stable model of $C$ which extend to the admissible cover. This group is non-trivial (of order two) precisely when the admissible cover contains an elliptic or hyperelliptic (genus 3 ) and the map to $L$ is two-to-one. Then the automorphism in question is the elliptic resp. hyperelliptic involution.

\section{Genus 4 fibrations}

The construction of gonal covers of the previous sections can be made explicit, complete with local models and discussion of the symplecticity of the branching divisor, in the case of Lefschetz fibrations with fiber genus $g=4$.

The generic gonality of genus 4 curves is 3 , with the trigonal covers arising from the canonical embedding of non-hyperelliptic curves of genus 4 in $\mathbb{P}^{3}$ as the transverse intersection of a quadric and a cubic surfaces. The trigonal structures are 2 if the quadric is nondegenerate, or just one if it is a cone (see [3, Ch. III]). Consequently, the Hurwitz space to use is $\tilde{H}=\overline{\mathcal{H}}_{3,4}$, and the map $h: \overline{\mathcal{H}}_{3,4} \rightarrow \overline{\mathcal{M}}_{4}$ is a branched $2: 1$ cover of $\overline{\mathcal{M}}_{4}$.

For this choice of genus, Corollary 9, Proposition 11 and Theorem 15 immediately yield as a particular case

Proposition 26 Let $f: X \rightarrow B$ be a symplectic Lefschetz fibration of curves of genus 4 over a closed oriented surface $B$. There exists an almost complex structure $J$ on $X$ compatible with the fibration and such that

(1) for A a union of suitably small, disjoint closed disks around the critical values of the fibration, $J$ is holomorphic on $f^{-1}(A)$. 
(2) the moduli map $\varphi: B \rightarrow \overline{\mathcal{M}}_{4}$ avoids the locus of curves with automorphisms in $\overline{\mathcal{M}}_{4}$ with the exception of the boundary divisor $\Delta_{1}$, and intersects transversely the divisors in $\overline{\mathcal{M}}_{4}$ which are image of boundary divisors of $\overline{\mathcal{H}}_{3,4}$ by $h: \overline{\mathcal{H}}_{3,4} \rightarrow \overline{\mathcal{M}}_{4}$, again with the exception of $\Delta_{1}$ which is intersected holomorphically with multiplicity 2.

Let us switch now from a plain Lefschetz fibration to one in which the trigonal structures can be glued: Consider the map $\varphi: B \rightarrow \overline{\mathcal{M}}_{4}$, which is holomorphic on $A$ and avoids the locus of curves with automorphisms in $B \backslash \AA$.

Notation Let $A_{1}$ be the union of the disks $B_{i} \subset A$ centered on critical values of $f$ whose singular fiber has moduli in $\Delta_{1}$.

The image $\varphi\left(S^{2} \backslash \AA_{1}\right)$ is contained in the Zariski open set $\overline{\mathcal{M}}_{4}^{\#}$ defined by curves with no automorphisms. Let

$$
\overline{\mathrm{C}}_{4}^{\#} \longrightarrow \overline{\mathcal{M}}_{4}^{\#}
$$

be the universal family of curves over the automorphism-free locus in the moduli space. By its universality property, our fibration $f: X \rightarrow B$ may be realised as the pull-back of this universal family by $\varphi$ :

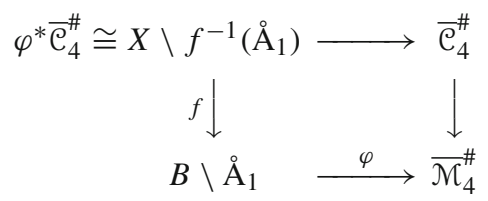

glued with the holomorphic family defined by $J$ over $A_{1}$. The identification $\varphi^{*} \overline{\mathrm{C}}_{4}^{\#} \cup f^{-1}\left(\AA_{1}\right) \cong$ $X$ is an isomorphism of smooth families of stable Riemann surfaces. Next, we pull-back the previous diagram via the morphism $h: \overline{\mathcal{H}}_{3,4} \rightarrow \overline{\mathcal{M}}_{4}$ to get the following one

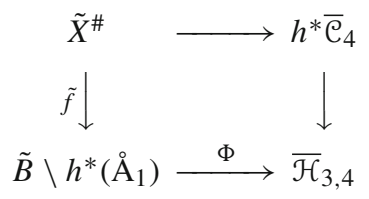

The proof of Theorem 25 shows that $h$ is generically smooth over $\Delta_{1}$ so the family $\tilde{X}^{\#}$ can be glued again to the holomorphic family over $A_{1}$. This is the family defined in Theorem 15

$$
\tilde{X} \stackrel{\tilde{f}}{\longrightarrow} \tilde{B}
$$

Proposition 27 The pullback fibration $\tilde{f}: \tilde{X} \rightarrow \tilde{B}$, with the almost complex structure induced by the pullback, is a symplectic Lefschetz fibration with genus 4 fibers over a closed orientable surface $\tilde{B}$ such that

(1) The induced map $\tilde{B} \rightarrow \overline{\mathcal{H}}_{3,4}$ avoids the locus formed by curves with automorphisms in $\mathcal{H}_{3,4}$, and intersects transversely the boundary divisors of $\partial \mathcal{H}_{3,4}$ with multiplicity \pm 1 , except for the divisors $D$ mapped by $h$ onto divisors of $\partial \mathcal{M}_{4}$, which are intersected with multiplicity 2 if $h(D)=\Delta_{1}$, and with multiplicity 1 in all other cases.

(2) The pullback from the universal family of trigonal curves defined over the Hurwitz space minus its locus of covers with automorphisms, glued to the holomorphic family over $h^{*} A_{1}$ defined by the almost complex structure $J$ of Proposition 26, defines a $\mathcal{C}^{\infty}$ 
family of spheres $p: P \rightarrow \tilde{B}$, holomorphic over the neighbourhoods $h^{*} A$ of singular fibers of the fibration $\tilde{f}$, such that $\tilde{f}$ factors through $p$ and a morphism

$$
\kappa: \tilde{X} \rightarrow P
$$

ramified along some singular locus $R \subset P$, and such that

- $\quad \kappa$ restricts to homolomorphic trigonal covers in the regular and irreducible singular fibers of $\tilde{f}$,

- $\quad \kappa$ is holomorphic in neighbourhoods of all singular fibers of $\tilde{f}$,

- in the reducible singular fibers $\tilde{X}_{s_{i}}$ of $\tilde{f}, \kappa$ restricts to a holomorphic mapping contracting one of the two components to a point, and mapping the other component with degree 2 or 3 to the Riemann sphere $P_{s_{i}}$,

Proof Consider the morphism $\pi: \overline{\mathcal{H}}_{3,4} \rightarrow \overline{\mathcal{M}}_{0,[12]}$ defined by sending each trigonal cover to its basis and branch locus, and the universal family $\overline{\mathcal{C}}_{0,[12]}^{\#}$ over $\overline{\mathcal{M}}_{0,[12]}^{\#}$, with superscript \# denoting again removal of the locus of covers with automorphisms. Denote $\tilde{B}^{\#}=\tilde{B} \backslash h^{*} A_{1}$. A family of spheres $\tilde{P}^{\#}$ is defined by the pullback

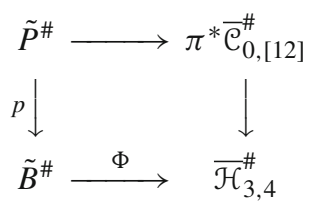

The restriction of the Lefschetz fibration $\tilde{f}$ to $h^{*} A_{1}$ also has, by Theorem 25 and Proposition 26 , a family of trigonal covers to stable genus 0 curves. This can be glued to $\tilde{P}^{\#} \rightarrow \tilde{B}^{\#}$ along the boundary $\partial A_{1}$, defining a family of spheres

$$
\tilde{P} \rightarrow \tilde{B}
$$

The fibers of $\tilde{P} \rightarrow \tilde{B}$ corresponding to curves over the boundary $\partial \mathcal{H}_{3,4}$ are reducible rational curves, with two components transversally meeting at one point.

Finally, notice that we also have a universal family of curves $\overline{\mathcal{u}}_{4}^{\#}$ over $\overline{\mathcal{H}}_{3,4}^{\#}$ obtained, from $h^{*} \overline{\mathcal{C}}_{4}^{\#}$ by blowing up those reducible fibres in $h^{*} \overline{\mathcal{C}}_{4}^{\#}$ so that they become the domain of an admissible covering. Thus we have a stabilization map $\overline{\mathcal{U}}_{4}^{\#} \rightarrow \tau^{*} \overline{\mathcal{C}}_{4}^{\#}$ and a trigonal covering map $\overline{\mathcal{U}}_{4}^{\#} \rightarrow \pi^{*} \overline{\mathcal{C}}_{0,[12]}^{\#}$. We have another pullback diagram

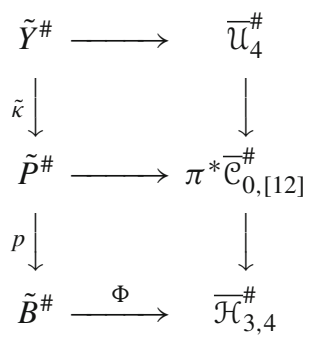

whose first column can be completed, applying Proposition 26 and the local model of admissible covers on the divisor $\partial_{3 \cup 1} \mathcal{H}_{3,4}$ mapped by $h$ onto $\Delta_{1}$, to morphisms

$$
\tilde{Y} \stackrel{\tilde{\kappa}}{\longrightarrow} \tilde{P} \stackrel{p}{\longrightarrow} \tilde{B}
$$


Let us denote the composition map $\tilde{g}=p \circ \tilde{\kappa}$. The stabilization map $\sigma: \tilde{Y} \rightarrow \tilde{X}$ corresponds to the blowing down of the $(-1)$-spheres in the fibres $\tilde{Y}_{s}$ of $\tilde{g}: \tilde{Y} \rightarrow \tilde{B}$ such that $\Phi(s) \in$ $\partial \mathcal{H}_{3,4}$. These are the $(-1)$-spheres required for the completion of the admissible triple cover (see [8]), and are listed in Definitions 17, 19, 21.

Finally, the family $\tilde{P} \rightarrow \tilde{B}$ is a smooth bundle of spheres, which is holomorphic around the singular values of $\tilde{f}$, with one point blown up over every value $s \in \tilde{B}$ such that $\Phi(s) \in$ $\partial \mathcal{H}_{3,4}$ to get the reducible basis for the corresponding admissible cover of Definitions 17 , 19, 21. Blowing down these $(-1)$-spheres defines the smooth bundle $P \rightarrow \tilde{B}$. The map $\tilde{X} \rightarrow \tilde{P} \rightarrow P$ is a trigonal cover at regular and irreducible singular fibers, but will contract to a point one of the irreducible components of reducible singular fibers and will have degree 2 or 3 on the other component acording to the list of covers from Definitions 17, 19, 21.

The generic fiber of the family of trigonal covers $\tilde{X} \rightarrow P \rightarrow \tilde{B}$ is a genus 4 curve covering 3:1 the Riemann sphere with 12 simple ramification points. The ramification points form a ramification locus $R \subset P$ for the map $\kappa: \tilde{X} \rightarrow P$. The singular covers in the family are given by the intersections of the image of the mapping $\Phi: \tilde{B} \rightarrow \overline{\mathcal{H}}_{3,4}$ with boundary divisors of Hurwitz space mapping to divisors in $\overline{\mathcal{M}}_{4}$. These divisors have been listed for any genus in Sect. 4, and the multiplicity with which they are intersected for even genus have been listed in Theorem 15. In the $g=4$ case this sums up as

Corollary 28 The trigonal fibration $\tilde{f}: \tilde{X} \rightarrow \tilde{B}$ of Proposition 27 has classifying map $\tilde{B} \rightarrow \overline{\mathcal{H}}_{3,4}$ intersecting the following boundary divisors of $\partial \overline{\mathcal{H}}_{3,4}$, with the indicated local models for the ramification locus $R \subset P$ over small disks $\mathbb{D}_{\epsilon} \subset \tilde{B}$ centered at the intersection points:

(1) $\partial_{\text {irr }} \mathcal{H}_{3,4}$, dominating $\Delta_{0}$ in $\partial \mathcal{M}_{4}$, with local model for $R$

$$
R_{s}=\left\{\left(z^{2}-s\right)\left(z-\lambda_{3}\right) \ldots\left(z-\lambda_{12}\right)=0\right\}
$$

(2) $\partial_{3 \cup 1,2} \mathcal{H}_{3,4}$, dominating $\Delta_{1}$ in $\partial \mathcal{M}_{4}$, with local model for $R$

$$
R_{s}=\left\{\left(z^{3}-s^{6}\right)\left(z-\lambda_{4}\right) \ldots\left(z-\lambda_{12}\right)=0\right\}
$$

(3) $\partial_{2 \cup 2,1} \mathcal{H}_{3,4}$, dominating $\Delta_{2}$ in $\partial \mathcal{M}_{4}$, with local model for $R$

$$
R_{s}=\left\{\left(z^{6}-s^{6}\right)\left(z-\lambda_{7}\right) \ldots\left(z-\lambda_{12}\right)=0\right\}
$$

(4) $\partial_{2} \cup 2,3 \mathcal{H}_{3,4}$, dominating $\Delta_{2}$ in $\partial \mathcal{M}_{4}$, with local model for $R$

$$
R_{s}=\left\{\left(z^{6}-s^{18}\right)\left(z-\lambda_{7}\right) \ldots\left(z-\lambda_{12}\right)=0\right\}
$$

$\partial_{e=2} \mathcal{H}_{3,4}$, with image a divisor in $\mathcal{M}_{4}$, and local model for $R$

$$
R_{s}=\left\{\left(z^{2}-s^{3}\right)\left(z-\lambda_{3}\right) \ldots\left(z-\lambda_{12}\right)=0\right\}
$$

where the local models for $R$ are given in coordinates $(s, z) \in p^{-1}\left(\mathbb{D}_{\epsilon}\right) \cong B(0, \epsilon) \times \mathbb{P}_{\mathbb{C}}^{1}$, and the parameters $\lambda_{j}$ are fixed constants.

The local models for the intersections with the divisors mapping onto $\partial \mathcal{M}_{4}$ are realised with the orientation given by the complex structure, whereas the local model for the intersection with the divisor $\partial_{e=2} \mathcal{H}_{3,4}$ may be realised with any orientation.

Proof The five possible intersections of $\tilde{B}$ with divisors in the boundary $\partial \mathcal{H}_{3,4}$ are those found in Sect. 4 for $(d, g)=(3,4)$. 
These intersections are realised in Sect. 4 as reducible covers $C \rightarrow L_{1} \cup L_{2}$, with the distribution of the 12 branch points of the generic cover $\tilde{X}_{s} \rightarrow P_{s} \cong \mathbb{P}_{\mathbb{C}}^{1}$ among $L_{1}, L_{2}$ as $(2,10),(3,9),(6,6),(6,6),(2,10)$ repectively in cases $(1)$ to $(5)$ of the statement.

The local models for the ramification locus $R$ around the reducible covers $C \rightarrow L_{1} \cup L_{2}$ are those listed in the proof of Theorem 25 for $(d, g)=(3,4)$.

The fact that local models around the divisors mapping onto the boundary $\partial \mathcal{M}_{4}$ preserve the complex orientation follows from the fact that the monodromy of the original Lefschetz fibration $f: X \rightarrow B$ is generated by positively oriented Dehn twists. The examples in the remark after Theorem 15 show that the orientation need not be preserved in the intersections of $\tilde{B}$ with $\partial_{e=2} \mathcal{H}_{3,4}$.

We are now interested in the pair $(P, R)$ given by the ramification locus of the map $\kappa$. We would like to attach a symplectic form $\omega_{P}$ to $P$ compatible with the map $p: P \rightarrow \tilde{B}$ and such that $R$ is symplectic with respect to $\omega_{P}$. We should clarify the condition that $R$ is symplectic with respect to $\omega_{P}$ in a singular point.

Definition 29 We say that the ramification locus $R \subset P$ is symplectic with respect to a symplectic form $\omega$ defined on $P$ whenever the restriction of $\omega$ to the tangent space $T_{p} R$ is non-degenerate in all the regular points $p \in R$ and, for singular $r \in R$, the restriction of $\omega$ to any limit

$$
\lim _{\text {smooth } p \rightarrow r} T_{p} R
$$

is non-degenerate.

Proposition 30 Let $p: P \rightarrow \tilde{B}$ be the family of spheres and $R \subset P$ the ramification locus from Proposition 27. Then,

(1) There is a finite set of values $\Delta \subset B$ such that $R \cap p^{-1}(B-\Delta)$ is smooth, and the restriction to it of $p$ is an unramified covering of $B-\Delta$ of degree 12. For each value in $\delta \in \Delta$ we have a local model for $R$ in a neighbourhood of the fibre $p^{-1}(\delta)$ which is described in Corollary 28.

(2) There exists a symplectic form $\omega_{P}$ on $P$ such that $p$ is a symplectic fibration and the ramification locus $R$ is symplectic with respect to $\omega_{P}$. This symplectic form admits a lift to a symplectic form $\omega_{\tilde{X}}$ on $\tilde{X}$ which is compatible with the map $\kappa: \tilde{X} \rightarrow P$.

Proof Part (1) follows from the construction of the sphere family $p: P \rightarrow \tilde{B}$ in the proof of Proposition 27: it is obtained by blowing down (-1)-curves at finitely many fibers of a family $\tilde{P} \rightarrow \tilde{B}$, which is itself a pullback of the universal family of bases of trigonal covers $\pi^{*} \overline{\mathcal{C}}_{0,[12]} \rightarrow \overline{\mathcal{H}}_{3,4}$. The ramification locus $R$ is by definition the pullback of the divisor of marked points in the universal curve $\overline{\mathcal{C}}_{0,[12]}$. This divisor is smooth, and a degree 12 unramified cover of the base outside the boundary $\partial \mathcal{H}_{3,4}$, which by Proposition 27 (1) intersects with the image $\Phi(\tilde{B})$ only at a finite set of points $\left\{b_{1}, \ldots, b_{m}\right\}=\Delta \subset \tilde{B}$, around which $R$ follows the local models of Corollary 28.

Part (2) is a slight extension of Thm. 2.1 in [15], from which we borrow the strategy for the proof: the locally trivial fibration $p: P \rightarrow S^{2}$, together with the local models of Corollary 28 around $p^{-1}(\Delta)$ provide us local symplectic forms. In the last case of Corollary 28 , if the holomorphic local model is satisfied with orientation opposite to the symplectic orientation of total space we will work with the complex conjugated local model, so that the orientation matches the global one and the ramification locus is antiholomorphic, thus still symplectic. 
Let us construct now a global symplectic form with the desired properties, using an argument adapted from the proof of Thurston's theorem [13].

First of all, we notice that the structure group of the fibration $p: P \rightarrow B$ may be reduced from $\operatorname{Diff}^{+}\left(S^{2}\right)$ to $\mathrm{SO}(3)$. Now let $b_{0} \in B$ be a base point and we fix a cohomology class $[\sigma] \in H^{2}\left(P_{b_{0}} ; \mathbb{Z}\right)$ where $\sigma$ is a symplectic form in $P_{b_{0}}:=p^{-1}\left(b_{0}\right)$. For each $b \in B$ let $\left[\sigma_{b}\right] \in H^{2}\left(P_{b} ; \mathbb{Z}\right)$ be the cohomology class obtained from $[\sigma]$ using a fixed parallel transport. (It is well defined, because the elements of the structure group of $p$ preserve the volume of a fibre.)

There exists a closed 2-form $\eta \in \Omega^{2}(P)$ such that, for every $b \in B$, satisfies $\left[i_{b}^{*} \eta\right]=\left[\sigma_{b}\right]$ where $i_{b}: P_{b} \hookrightarrow P$ is the inclusion. We recall that Corollary 28 furnishes analytic local models for $p$ and the ramification locus over discs $U_{1}, \ldots, U_{m}$, centered at $b_{1}, \ldots, b_{m}$ respectively. Now let $\left\{U_{\alpha}\right\}_{\alpha}$ be a simple covering of $B$ (containing the discs $U_{1}, \ldots, U_{m}$ ), where $U_{\alpha}$ are open discs such that $b_{i} \notin \bar{U}_{\alpha}$ when $\alpha \neq i$.

For each $U_{i}(i=1, \ldots, m)$ we endow a symplectic form $\omega_{i}$ in $P_{i}=p^{-1}\left(U_{i}\right)$ in such a way that represents the class $[\sigma]$ in the fibres and that the ramification locus $R_{i} \subset P_{i}$ is symplectic with respect to it (see the remark above).

This can be accomplished using the local model for $R_{i} \subset P_{i} \rightarrow U_{i}$. Since the local model is analytic there exists a Kähler form on $P_{i}$ compatible with the fibration, and due to the fact that the model for the ramification locus $R_{i}$ is complex or complex-conjugate, the restriction of the Kähler form to $R_{i}$ is a symplectic form. By rescaling the Kähler form, we get a symplectic form $\omega_{i}$ on $P_{i}$ compatible with the fibration, representing $[\sigma]$ in the fibres, and such that $R_{i}$ is symplectic with respect to $\omega_{i}$.

For the rest of the $U_{\alpha}$ we perform the following construction. The fibration $P_{\alpha} \rightarrow U_{\alpha}$ is equivalent to the trivial one, so we furnish the former with the symplectic form $\omega_{\alpha}$ coming from the later $U_{\alpha} \times S^{2} \rightarrow U_{\alpha}$ rescaled so that $\omega_{\alpha}$ represents $[\sigma]$ on the fibres. i.e.

Since every $U_{\alpha}$ is contractible, the two 2-forms $\eta$ and $\omega_{\alpha}$ are cohomologous in $p^{-1}\left(U_{\alpha}\right)$,

$$
\omega_{\alpha}-\eta=d \lambda_{\alpha}, \quad \text { where } \lambda_{\alpha} \in \Omega^{1}\left(p^{-1} U_{\alpha}\right) .
$$

Now we choose $\left\{\rho_{\alpha}\right\}_{\alpha}$ a partition of unity subordinate to the covering $\left\{U_{\alpha}\right\}_{\alpha}$ such that for each $b_{i}, i=1, \ldots, n$, the function $\rho_{i}$ has constant value 1 on $U_{i}$. We then define

$$
\tilde{\omega}=\eta+\sum_{\alpha} d\left(\left(\rho_{\alpha} \circ p\right) \lambda_{\alpha}\right) .
$$

Then $\tilde{\omega}$ is a closed 2-form in $P$ compatible with $p$, representing $[\sigma]$ in each fibre of the family $p: P \rightarrow B$, and that coincides with $\omega_{i}$ in $U_{i}$. The form $\tilde{\omega}$ need not to be a symplectic form on $P$, but it is non-degenerate on the tangent spaces to the fibres, so that the fibres are symplectic with respect to $\tilde{\omega}$. At this point we use Thurston's argument, which asserts that the 2-form

$$
\omega_{K}=\tilde{\omega}+K \cdot p^{*} \omega_{B}
$$

is a symplectic form on $P$ for $K>0$ large enough. (Here $\omega_{B}$ stands for a symplectic form on the base $B$.)

We notice the fact that, in each of the open $U_{i}$, we can add to $\omega_{i}$ any multiple of $p^{*}\left(\omega_{B \mid U_{i}}\right)$ and the resulting 2 -form is Kähler, and by the argument of a previous paragraph, the restricition to $R_{i}$ is a symplectic form. This shows that the restriction of $\omega_{K}$ to $R_{i}$ is symplectic for any $K>0$.

In order to complete the proof we need to show that $R$ is symplectic with respect to $\omega_{K}$ for some $K$. If $q \in R$ is a not a critical point of the composition $p_{\mid R}: R \hookrightarrow P \rightarrow B$ then there 
is a neighbourhood of $q$ in $R$ that the restriction of $\omega_{K}$ to it is non degenerate for $K$ large enough (use an argument like Thurston's one). The case that $q$ is a critical point is one of our local models and is covered in the previous paragraph; which assures that $\omega_{K}$ is symplectic in some neighbourhood of $q$ for any $K>0$. Finally, by compactness of $R$ there is a $K>0$ large enough such that $R$ is symplectic with respect to $\omega_{K}$.

Acknowledgments This work has had a protracted development over several years, and has benefited from discussions of the authors with many people, specially D. Auroux, M.A. Barja, D. Kotschick, E. Miranda, V. Muñoz, B. Siebert, and Gang Tian. It has also benefited greatly from the careful scrutiny of an anonymous referee. Universidad Autónoma de Madrid provided hospitality to two of the authors during early stages of the project.

\section{References}

1. Amorós, J., Bogomolov, F., Katzarkov, L., Pantev, T.: Symplectic Lefschetz fibrations with arbitrary fundamental groups. J. Differ. Geom. 54, 489-545 (2000)

2. Auroux, D.: Fiber sums of genus 2 Lefschetz fibrations. Turkish J. Math. 27, 1-10 (2003)

3. Arbarello, E., Cornalba, M., Griffiths, P.A., Harris, J.: Geometry of Algebraic Curves, vol. I. Grundlehren der mathematischen Wissenschaften, vol. 267 Springer, (1985)

4. Arbarello, E., Cornalba, M., Griffiths, P.A.: Geometry of Algebraic Curves, vol. II. Grundlehren der mathematischen Wissenschaften, vol. 268 (2010)

5. Berna Sepúlveda, I.: Monodromías geométricas en familias de curvas de género 4. Doctoral dissertation. UPC, 2012. http://www.tdx.cat/handle/10803/91684

6. Fulton, W.: Hurwitz schemes and irreducibility of moduli of algebraic curves. Ann. Math. 90, 542$575(1969)$

7. Gompf, R.E., Stipsicz, A.I.: 4-Manifolds and Kirby Calculus. Graduate Studies in Mathematics, vol. 20. American Mathematical Society, Providence, RI (1999)

8. Harris, J., Morrison, I.: Moduli of Curves, GTM 187. Springer, New York (1998)

9. Harris, J., Mumford, D.: On the Kodaira dimension of moduli space of curves, with an appendix by William Fulton. Invent. Math. 67, 23-88 (1982)

10. Husemoller, D.: Fibre Bundles, GTM 20. Springer, New York (1994)

11. Kas, A.: On the handlebody descomposition associated to a Lefschetz fibration. Pac. J. Math. 89, 89-104 (1980)

12. McDuff, D., Salamon, D.: J-Holomorphic Curves and Quantum Cohomology. University Lecture Series, vol. 6. American Mathematical Society, Providence, RI (1994)

13. McDuff, D., Salamon, D.: Introduction to Symplectic Topology. Oxford Mathematical Monographs. Oxford University Press, New York (1995)

14. Mochizuki, S.: The Geometry of the compactification of the Hurwitz scheme. Publ. RIMS Kyoto 31, 355441 (1995)

15. Siebert, B., Tian, G.: On hyperelliptic symplectic Lefschetz fibrations of four manifolds. Commun. Contemp. Math. 1, 255-280 (1999)

16. Siebert, B., Tian, G.: On the holomorphicity of genus two Lefschetz fibrations. Ann. Math. 161, 9591020 (2005) 